Supporting Information

\title{
An Open-shell, Luminescent, Two-dimensional Coordination Polymer with a Honeycomb Lattice and Triangular Organic Radical
}

Shun Kimura, Motoyuki Uejima, Wataru Ota, Tohru Sato, Shinpei Kusaka,

Ryotaro Matsuda, Hiroshi Nishihara, ${ }^{*}$ and Tetsuro Kusamoto* 


\section{Table of Contents}

Materials and methods $\quad$ S3

$\begin{array}{ll}\text { Synthesis } & \text { S6 }\end{array}$

Theoretical calculations $\quad$ S9

Table S1 Hyperfine coupling constants used for ESR simulation and DFT-calculated values $\quad$ S15

Table S2 Absolute emission quantum yields and luminescent lifetimes of trisPyM in various solventsS15

Table S3 Half-lives and absorption coefficients of trisPyM and comparisons of photostability $\quad$ S16

Table S4The photophysical parameters of radicals in $\mathrm{CH}_{2} \mathrm{Cl}_{2}$

Table S5 Crystallographic data of trisPyM and trisZn $\quad$ S17

Figure S5. ESR signals of trisPyM $\quad$ S18

Figure S6. Cyclic voltammogram of trisPyM $\quad$ S18

Figure S7. Absorption and emission spectra of trisPyM in various solvents $\quad$ S18

Figure S8. Excitation spectrum and absorption spectrum of trisPyM $\quad$ S19

Figure S9. Energies of the longest absorption band peaks and the emission band peaks of radicals S19

Figure S10. Emission decays of trisPyM and trisZn in the solid states at $79 \mathrm{~K}$

Figure S11. Enlarged figure of Figure $5 \quad$ S20

Figure S12. A photograph of crystals of trisZn $\quad$ S21

Figure S13. Thermogravimetric analysis (TGA) curves of trisZn and trisPyM $\quad$ S21

Figure S14. ${ }^{1} \mathrm{H}$ NMR spectra of trisZn in acetone-d6.

Figure S15. Powder X-ray diffraction patterns of trisZn $\quad$ S22

Figure S16. Temperature dependence of the emission spectra of trisZn $\quad$ S23

Figure S17. Emission spectra of trisZn at $79 \mathrm{~K}$ for the as-prepared sample and the sample after evacuation at $60^{\circ} \mathrm{C}$ for 12 hours $\quad$ S23

Figure S18. Absorption spectra of trisPyM and trisZn before and after UV light irradiation. S22

Figure S19. Emission spectra of trisPyM and trisZn at $79 \mathrm{~K}$ before and after UV light irradiation. S24

Figure S20. Magnetic properties of trisZn $\quad$ S24

Figure S21. ESR signals of trisPyM and trisZn in the solid states $\quad$ S25

$\begin{array}{ll}\text { References } & \text { S26 }\end{array}$ 


\section{Materials}

All chemicals were reagent grade and purchased commercially. Water was purified using AUTOPURE WD500 (Yamato Scientific Co., Ltd.). Acetonitrile ( $\mathrm{MeCN})$, dichloromethane $\left(\mathrm{CH}_{2} \mathrm{Cl}_{2}\right)$, diethyl ether $\left(\mathrm{Et}_{2} \mathrm{O}\right), n$-hexane, tetrahydrofuran $(\mathrm{THF})$ and toluene used for syntheses and measurements were purified through organic solvent purifier (Nikko Hansen Co., Ltd.). TTM, PyBTM, and bisPyTM were prepared by following procedures reported in the literature. ${ }^{1,2}$

\section{Equipments}

${ }^{1} \mathrm{H}$ and ${ }^{13} \mathrm{C}$ spectra were recorded with a JEOL JNM-ECS400 spectrometer at room temperature. Chemical shifts in ppm were referenced to tetramethylsilane $(0.00 \mathrm{ppm})$ as an internal standard. UV-vis absorption spectra were recorded with a JASCO V570 spectrophotometer and a JASCO V770 spectrophotometer. Steady-state emission and excitation spectra at room temperature were measured with JASCO FP8600 and FP8600K spectrometers. Sample solutions were bubbled with argon before measurement. Absolute photoluminescence quantum yields of solution samples were measured with a Hamamatsu Photonics C9920-02G. Fluorescence lifetime measurements of solution samples were performed using a Hamamatsu Photonics Quantaurus-Tau C11367-02. Emission spectra and fluorescence lifetime measurements at $79 \mathrm{~K}$ were performed using a measurement system with a picosecond diode laser with the emission wavelength of $375 \mathrm{~nm}$ (Advanced Laser Diode Systems PIL037X) as light source, a single grating spectrometer (Andor Kymera193i-B1), and a multichannel CCD detector (Andor iDus DV420A-OE). ESR spectra were recorded with a JOEL JES-FA200 spectrometer or Bruker EMX Plus spectrometer. 4-Hydroxy-TEMPO was used as a standard for estimating spin concentration. Observed $g$-values were calibrated with $\mathrm{Mn}^{2+} / \mathrm{MgO}$ marker. The magnetic susceptibilities were measured on Quantum Design MPMS-7 SQUID magnetometers. Cyclic voltammetry was carried out under an argon atmosphere with a GC working electrode, a platinum wire counter electrode and an $\mathrm{Ag}^{+} / \mathrm{Ag}$ reference electrode (10 mM AgClO 4 and $0.1 \mathrm{mM}^{n} \mathrm{Bu}_{4} \mathrm{NClO}_{4}$ in $\mathrm{MeCN}$ solution) with an ALS-650DT voltammetric analyzer. The platinum working electrode was polished with alumina abrasive and washed with water and acetone with ultrasonication. The counter electrode was heated with oxygen flame to remove organic compounds on the surface and washed with acetone. The reference electrode was washed with acetone. The potential of the voltammograms is shown relative to the $\mathrm{Fc}^{+} / \mathrm{Fc}$ redox couple. Powder X-ray diffraction measurements were performed at room temperature using a Panalytical

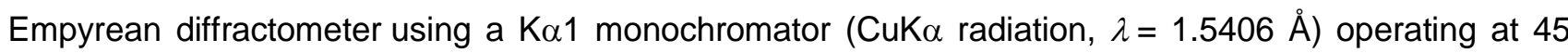
$\mathrm{kV} / 40 \mathrm{~mA}$.

\section{Single crystal X-ray crystallography}

The data for single crystal X-ray diffraction analysis were collected at $143 \mathrm{~K}$ for trisPyM and 123 $\mathrm{K}$ for trisZn on a ROD, Synergy Custom system (Rigaku Oxford Diffraction) equipped with mirror monochromated Mo-Ka radiation. A suitable single crystal was mounted on a looped film (micromount) with Paraton-N. Data were processed using CrysAlisPro 1.171.39.43c (Rigaku Oxford Diffraction). The 
structure was solved using ShelXT ${ }^{3}$ and the whole structure was refined against $F^{2}$ with SHELXL-2018/3. ${ }^{4}$ All non-hydrogen atoms were refined anisotropically. Hydrogen atoms were located in idealized positions and were refined using a riding model with fixed thermal parameters. Crystal structure data (CIF, CCDC 2041203 and 2048812) can be obtained free of charge from The Cambridge Crystallographic Data Centre.

\section{Computational details}

DFT calculations were executed using the Gaussian16 program package. ${ }^{5}$ The geometries of the compounds were optimized without symmetry constraints. Calculations were performed using the unrestricted Becke three-parameter hybrid functional with Lee-Yang-Parr correlation functional (B3LYP) ${ }^{6}$ with the $6-31 G(d, p)$ basis set. ${ }^{7}$ Frequency calculations were carried out to ensure that the optimized geometries were minima on the potential energy surface, in which no imaginary frequencies were observed in any of the compounds. TDDFT calculations were performed using UB3LYP to calculate the first 20 doublet transitions. Solvent $\left(\mathrm{CH}_{2} \mathrm{Cl}_{2}\right)$ effect was considered using the PCM model. ${ }^{8} \mathrm{VCC}$ and VCDs were calculated using our in-house codes.

\section{Evaluation of stability of trisPyM in solutions under UV light}

A solution (ca. $5 \times 10^{-6} \mathrm{M}, 2.0 \mathrm{~mL}$ ) in 1-cm-optical-path-length quartz cells was bubbled with argon, sealed, and set at a JASCO FP8600 spectrometer. Intensity of luminescence at 700, 650, 585, and 585 $\mathrm{nm}$ was monitored exciting at 350, 355, 370, and $370 \mathrm{~nm}$ light (excitation slit was $20 \mathrm{~nm}$, and shutter control was off), for trisPyM, bisPyTM, PyBTM, and TTM respectively. Logarithm of fluorescence intensity versus time was plotted and a slope of approximate line was estimated to be a rate of photolysis.

\section{Evaluation of stability of trisPyM and trisZn in the solid states under UV light}

At room temperature: a solid-state sample was set at a JASCO FP8600 spectrometer and irradiated with $350 \mathrm{~nm}$ light (excitation slit was $20 \mathrm{~nm}$, and shutter control was off). The sample was dissolved in $\mathrm{CH}_{2} \mathrm{Cl}_{2}$ and ethyl acetate for trisPyM and trisZn, respectively, and the absorption spectra before and after continuous UV light irradiation $\left(\lambda_{\mathrm{ex}}=350 \mathrm{~nm}\right.$ ) were compared. At $79 \mathrm{~K}$ : emission spectra and intensities of luminescence at 667 and $695 \mathrm{~nm}$ was monitored with exciting at $375 \mathrm{~nm}$ light $(5 \mathrm{MHz}$, tune value: $25 \%$ ) for trisPyM and trisZn, respectively. The system for the measurements is same as that used for emission spectra and fluorescence lifetime measurements at $79 \mathrm{~K}$ described above.

\section{Estimations of luminescent quantum yields of trisPyM and trisZn in the solid states}

Absolute emission quantum yields of trisPyM and trisZ $\mathbf{n}$ in the emission wavelength range of 500 to $800 \mathrm{~nm}$ were measured using a JASCO FP8500 spectrometer equipped with an ILFC-847 liquid $\mathrm{N}_{2}$ cooled integrating sphere. The quantum yields were $3.3 \%$ and $1.8 \%$ upon excitation at $\lambda=370 \mathrm{~nm}$, respectively. The emission quantum yields in the emission wavelength range of 500 to $900 \mathrm{~nm}$ were calculated based on the peak areas of emission bands to be $3.4 \%$ and $2.0 \%$, respectively. 


\section{Gas adsorption measurement of trisZn}

Gas adsorption measurements were performed on a microtrac-MRB BELSORP-mini volumetric gas adsorption analyzer. The sample of trisZn was activated under dynamic vacuum at $60^{\circ} \mathrm{C}$ for $12 \mathrm{~h}$ prior to the measurement. Grade $1 \mathrm{~N}_{2}$ and $\mathrm{CO}_{2}$ gases were used (>99.99995 vol.\% and >99.995 vol.\%, respectively). The measurement temperature was controlled by liquid bath (liquid $\mathrm{N}_{2}$ for $\mathrm{N}_{2}$ adsorption at $77 \mathrm{~K}$ and dry ice in methanol for $\mathrm{CO}_{2}$ adsorption at $195 \mathrm{~K}$ ). 


\section{Synthesis}

trisPyM was synthesized following Scheme S1. Nucleophilic addition reactions of 1,3,5-trichlorobenzene in acidic conditions have been employed for the preparations of the precursors of TTM, ${ }^{9}$ PyBTM, ${ }^{1}$ and bisPyTM. ${ }^{2}$ However, direct introductions of third 3,5-dichloropyridine moiety for preparations of $\alpha H$-trisPyM did not proceed by a nucleophilic addition in acidic conditions of 3,5-dichloropyridine because of the electron deficiency of the pyridine rings. I therefore adopted the synthetic route, with the chlorination reaction after the construction of the (3-chloro-4-pyridyl)bis(3,5-dichloro4-pyridyl)methane.
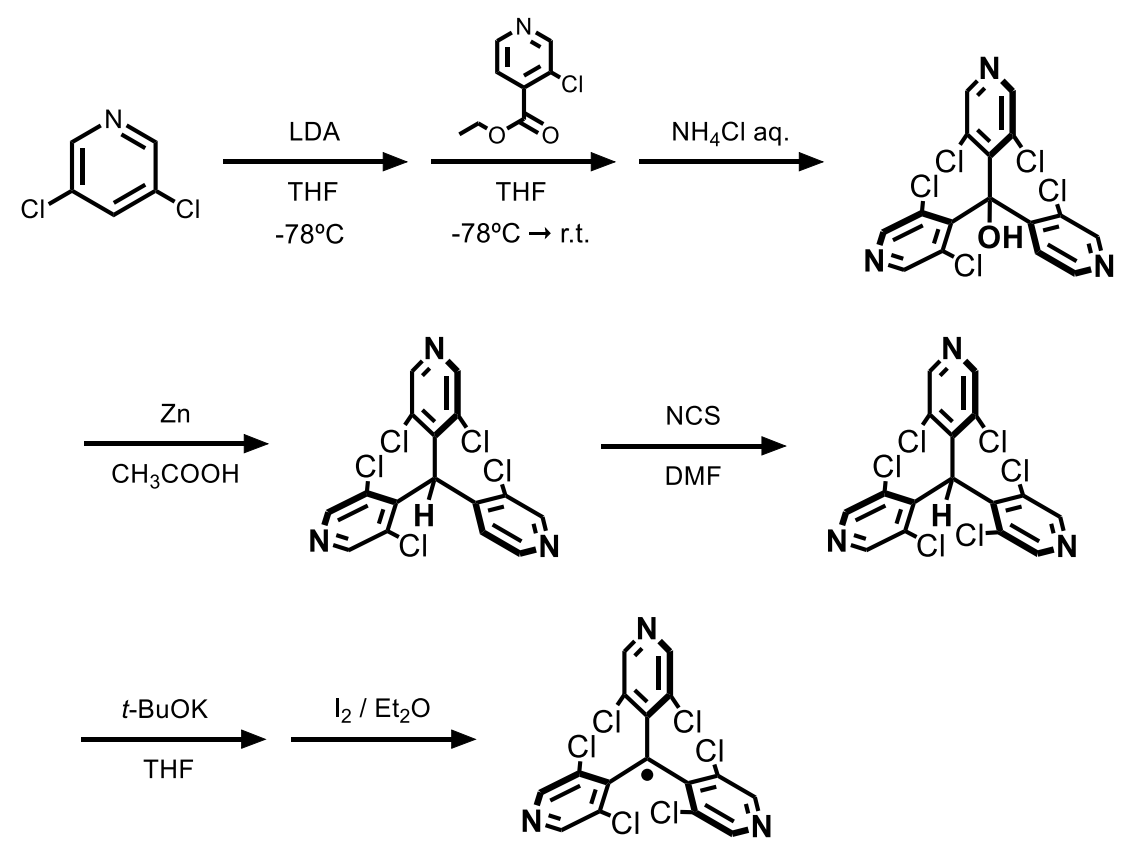

Scheme S1. Synthetic route of trisPyM.

\section{Synthesis of (3-chloro-4-pyridyl)bis(3,5-dichloro4-pyridyl)methanol}

Under a nitrogen atmosphere, a solution of diisopropylamine $(10 \mathrm{~mL}, 71 \mathrm{mmol})$ in dry THF $(60 \mathrm{~mL})$ was cooled to $-78^{\circ} \mathrm{C}$ (acetone/dry ice bath) and was added a $1.60 \mathrm{M} \mathrm{n}$-BuLi ( $45 \mathrm{~mL}, 72 \mathrm{mmol}$ ) in hexane. Obtained solution of lithium diisopropylamide was heated to room temperature in 15 minutes and cooled to $-78^{\circ} \mathrm{C}$ again. To the solution was added a solution of 3,5-dichloropyridine $(9.76 \mathrm{~g}, 66 \mathrm{~mol})$ in dry THF $(60 \mathrm{~mL})$. A solution of ethyl 3chloroisonicotinate $(5.2 \mathrm{~g}, 28 \mathrm{mmol})$ in dry THF $(85 \mathrm{~mL})$ was added dropwise and the mixture was left in the cooling bath to reach the room temperature. After addition of saturated aqueous $\mathrm{NH}_{4} \mathrm{Cl}$, the mixture was extracted with ethyl acetate. The organic layer was separated and the water layer was washed with $\mathrm{Et}_{2} \mathrm{O}$ (100 $\mathrm{mL} \times 3$ ). The organic phase was collected, dried $\left(\mathrm{Na}_{2} \mathrm{SO}_{4}\right)$, and filtered. Evaporation of the solvent left a black residue. The residue was purified with $\mathrm{SiO}_{2}$ column chromatography (ethyl acetate/ $n$-hexane $=1 / 1$ ) to give a white solid of the product $\left(4.60 \mathrm{~g}, 10.6 \mathrm{mmol} 38 \%\right.$ yield). ${ }^{1} \mathrm{H}$ NMR (Acetone-d6, $\left.400 \mathrm{MHz}\right): \delta=6.20(\mathrm{~s}, 1 \mathrm{H}$, $\mathrm{OH}), 7.00(\mathrm{~d}, 1 \mathrm{H}, J=5.2 \mathrm{~Hz}), 8.47(\mathrm{~d}, 1 \mathrm{H}, J=5.2 \mathrm{~Hz}), 8.55(\mathrm{~s}, 4 \mathrm{H}), 8.51(\mathrm{~s}, 1 \mathrm{H}), 8.66(\mathrm{~s}, 1 \mathrm{H})$ Although a small 
unassignable peak at $\delta=8.51$ (s) was also observed, this product was used in the next step without further purification. FAB-MS: $m / z=436\left(\left[\mathrm{C}_{16} \mathrm{H}_{9} \mathrm{~N}_{3} \mathrm{OCl}_{5}\right]^{+}\right)$Elem. Anal. Calcd for $\mathrm{C}_{16} \mathrm{H}_{8} \mathrm{~N}_{3} \mathrm{OCl}$ : C 44.13, $\mathrm{H} 1.85, \mathrm{~N}$ 9.65. Found, C 44.20, H 2.05, N 9.30.

\section{Synthesis of (3-chloro-4-pyridyl)bis(3,5-dichloro-4-pyridyl)methane}

Under a nitrogen atmosphere, to a solution of (3-chloro-4-pyridyl)bis(3,5-dichloro-4-pyridyl)methanol (4.69 g, $10.7 \mathrm{~mol})$ in glacial acetic acid $(126 \mathrm{~mL})$ was added a zinc powder $(10.5 \mathrm{~g})$, and was vigorously stirred for 7 days. The mixture was quenched with aqueous $\mathrm{Na}_{2} \mathrm{CO}_{3}$ and extracted with ethyl acetate. The organic layer was separated and the water layer was washed with ethyl acetate $(100 \mathrm{~mL} \times 3)$. The organic phase was collected, dried $\left(\mathrm{Na}_{2} \mathrm{SO}_{4}\right)$, and filtered. Evaporation of the solvent left a pale-yellow solid. The solid was purified with $\mathrm{SiO}_{2}$ column chromatography (ethyl acetate $/ n$-hexane $=1 / 1)$ to give a white solid of the product $(1.79 \mathrm{~g}, 4.3 \mathrm{mmol}$, $40 \%$ yield). ${ }^{1} \mathrm{H} \mathrm{NMR}\left(\mathrm{CDCl}_{3}, 400 \mathrm{MHz}\right): \delta=6.59(\mathrm{~s}, 1 \mathrm{H}), 6.79(\mathrm{~d}, 1 \mathrm{H}, J=5.2 \mathrm{~Hz}), 8.42(\mathrm{~d}, 1 \mathrm{H}, J=5.2 \mathrm{~Hz}), 8.52$ (s, 4H), $8.64(\mathrm{~s}, 1 \mathrm{H}){ }^{13} \mathrm{C}$ NMR $\left(\mathrm{CDCl}_{3}, 100 \mathrm{MHz}\right): \delta 48.52,125.13,132.62,133.60,140.93,142.67,148.20$, 149.00, 149.45 GC-MS: $m / z=419\left(\left[\mathrm{C}_{16} \mathrm{H}_{8} \mathrm{~N}_{3} \mathrm{Cl}_{5}\right]^{+}\right)$Elem. Anal. Calcd for $\mathrm{C}_{16} \mathrm{H}_{8} \mathrm{~N}_{3} \mathrm{Cl}_{5}: \mathrm{C}$ 45.81, H 1.92, N 10.02. Found, C 45.84, H 2.15, N 9.82.

\section{Synthesis of tris(3,5-dichloro-4-pyridyl)methane ( $\alpha H$-trisPyM)}

Under a nitrogen atmosphere, a solution of (3-chloro-4-pyridyl)bis(3,5-dichloro-4-pyridyl)methane (903 mg, $2.15 \mathrm{mmol}$ ) and $\mathrm{N}$-chlorosuccinimide (NCS) $(3.0 \mathrm{~g}, 22 \mathrm{mmol}$ ) in anhydrous $\mathrm{N}, \mathrm{N}$-dimethylformamide $(50 \mathrm{~mL})$ was heated to $150^{\circ} \mathrm{C}$. The mixture was stirred for 1 hour at $150^{\circ} \mathrm{C}$, and then was quenched with water. The resulting mixture was extracted with ethyl acetate. The organic layer was separated and the water layer was washed with ethyl acetate $(100 \mathrm{~mL} \times 3)$. The organic phase was collected, dried $\left(\mathrm{Na}_{2} \mathrm{SO}_{4}\right)$, and filtered. Evaporation of the solvent left a brown residue. The residue was purified with twice $\mathrm{SiO}_{2}$ column chromatography (eluent: $\mathrm{CH}_{2} \mathrm{Cl}_{2}$ for the first time and ethyl acetate $/ n$-hexane $=1 / 2$ for the second time) to give a white solid of the product ( 512 $\mathrm{mg}, 1.13 \mathrm{mmol}, 53 \%$ yield). ${ }^{1} \mathrm{H}$ NMR $\left(\mathrm{CDCl}_{3}, 400 \mathrm{MHz}\right): \delta 6.71(\mathrm{~s}, 1 \mathrm{H}), 8.42(\mathrm{~s}, 3 \mathrm{H}), 8.55(\mathrm{~s}, 3 \mathrm{H}){ }^{13} \mathrm{C}$ NMR ( $\left.\mathrm{CDCl}_{3}, 100 \mathrm{MHz}\right): \delta 49.16,133.47,134.17,141.18,147.92,149.57 \mathrm{GC}-\mathrm{MS}: \mathrm{m} / \mathrm{z}=453\left(\left[\mathrm{C}_{16} \mathrm{H}_{7} \mathrm{~N}_{3} \mathrm{Cl}_{6}\right]^{+}\right)$Elem. Anal. Calcd for $\mathrm{C}_{16} \mathrm{H}_{7} \mathrm{~N}_{3} \mathrm{Cl}_{6}$ : C 42.33, H 1.55, N 9.26. Found, C 42.07, H 1.67, N 9.15.

\section{Synthesis of tris(3,5-dichloro-4-pyridyl)methyl radical (trisPyM) ${ }^{1}$}

Under a nitrogen atmosphere, $\alpha \mathrm{H}$-trisPyM $(910 \mathrm{mg}, 2.02 \mathrm{mmol})$ was dissolved in dry THF $(200 \mathrm{~mL}) .1 \mathrm{M}$ solution of $t$-BuOK in THF $(10 \mathrm{~mL})$ was added dropwise. The reaction mixture was stirred overnight in the dark. $\mathrm{I}_{2}(2.80 \mathrm{~g}, 11 \mathrm{mmol})$ in dry $\mathrm{Et}_{2} \mathrm{O}(380 \mathrm{~mL})$ was added and stirred for $3 \mathrm{~h}$. Remaining $\mathrm{I}_{2}$ was reduced by washing with $10 \% \mathrm{Na}_{2} \mathrm{~S}_{2} \mathrm{O}_{3}$ aq. 3 times. The water layer was extracted with $\mathrm{Et}_{2} \mathrm{O}$ once, and the combined organic layer was dried with $\mathrm{Na}_{2} \mathrm{SO}_{4}$. The red solution was filtered, evaporated, purified by $\mathrm{Al}_{2} \mathrm{O}_{3}$ column chromatography $\left(\mathrm{CH}_{2} \mathrm{Cl}_{2} / n\right.$-hexane $\left.=1 / 1\right)$ and dried in vacuo to afford trisPyM $(740 \mathrm{mg}, 1.63 \mathrm{mmol}, 81 \%)$ as a red solid. HRMS (negative ion mode ESI-TOF) $\mathrm{m} / \mathrm{z}$ : [M]- Calcd for $\mathrm{C}_{16} \mathrm{H}_{6} \mathrm{~N}_{3} \mathrm{Cl}_{6}$ 449.8698; Found 449.8682 Elem. Anal. Calcd for 
$\mathrm{C}_{16} \mathrm{H}_{6} \mathrm{~N}_{3} \mathrm{Cl}_{6}$ : C 42.43, H 1.34, N 9.28. Found, C 41.97, H 1.49, N 9.10. ESR: Spin concentration of trisPyM in $\mathrm{CH}_{2} \mathrm{Cl}_{2}\left(1.2 \times 10^{-5} \mathrm{M}\right)$ was estimated by comparing the value of twice-integration of the signal intensity with that of the reference sample (4-hydroxy-TEMPO in $\mathrm{CH}_{2} \mathrm{Cl}_{2} ; 1.5 \times 10^{-5} \mathrm{M}$ ). The existence of $S=1 / 2$ spin on one trisPyM molecule was confirmed.

\section{Synthesis of $\left[\left[\mathrm{Zn}^{\prime \prime}(\mathrm{hfac})_{2}\right]_{3}[\operatorname{trisPyM}]_{2}\right]_{\mathrm{n}}$ (trisZn)}

A solution of $\mathrm{Zn}^{\prime \prime}(\mathrm{hfac})_{2} \cdot 2 \mathrm{H}_{2} \mathrm{O}(14.9 \mathrm{mg}, 0.029 \mathrm{mmol})$ and trisPyM (8.5 mg, $\left.0.019 \mathrm{mmol}\right)$ dissolved in dry $\mathrm{CHCl}_{3}(10 \mathrm{~mL})$ was slowly evaporated under a $n$-hexane atmosphere. The obtained red crystals of trisZn were washed with $n$-hexane, dried in vacuo, and characterized as $\left[\left[\mathrm{Zn}^{\prime \prime}(\mathrm{hfac})_{2}\right]_{3}[\text { trisPyM}]_{2}\right]_{\mathrm{n}}(13.1 \mathrm{mg})$ in $59 \%$ yield. Elem. Anal. Calcd for $\mathrm{C}_{31} \mathrm{H}_{9} \mathrm{Cl}_{6} \mathrm{~F}_{18} \mathrm{~N}_{3} \mathrm{O}_{6} \mathrm{Zn}_{1.5}: \mathrm{C} 31.77, \mathrm{H}$ 0.77, N 3.58. Found: C 31.45, H 0.86, N 3.73. Solid-state ESR: Spin concentration was estimated by comparing the value of twice-integration of the signal intensity with that of the reference sample (4-hydroxyTEMPO) in the solid state. The existence of two $S=1 / 2$ spin on $\left[\mathrm{Zn}^{\prime \prime}(\mathrm{hfac})_{2}\right]_{3}[\mathrm{trisPyM}]_{2}$ unit was confirmed. 


\section{Theoretical calculations}

\section{Rate Constant of Internal Conversion}

Within the crude adiabatic approximation, ${ }^{10}$ the vibronic state $\left|\Phi_{i m}(\mathbf{r}, \mathbf{Q})\right\rangle$ is expressed as the product of vibrational $\left|\chi_{i m}(\mathbf{Q})\right\rangle$ and electronic states $\left|\Psi_{i}\left(\mathbf{r} ; \mathbf{R}_{\mathbf{0}}\right)\right\rangle$ fixed at nuclear configuration $\mathbf{R}_{\mathbf{0}}$; that is $\left|\Phi_{i m}(\mathbf{r}, \mathbf{Q})\right\rangle=$ $\left|\chi_{i m}(\mathbf{Q})\right\rangle\left|\Psi_{i}\left(\mathbf{r} ; \mathbf{R}_{\mathbf{0}}\right)\right\rangle$, where $\mathbf{r}$ is a set of electronic coordinates and $\mathbf{Q}$ is a set of mass-weighted normal coordinates. Then, the rate constant of internal conversion from electronic states $i$ to $j$ is given by ${ }^{11}$

$$
k_{i \rightarrow j}^{\mathrm{lC}}=\frac{2 \pi}{\hbar} \sum_{m n} P_{i m}(T) \sum_{\alpha}\left|V_{i j, \alpha}\right|^{2}\left|\left\langle\chi_{i m}(\mathbf{Q})\left|Q_{\alpha}\right| \chi_{j n}(\mathbf{Q})\right\rangle\right|^{2} \delta\left(E_{i m}-E_{j n}\right) .
$$

Here, $P_{i m}(T)$ is the Boltzmann distribution function of the initial vibronic state at temperature $T . V_{i j, \alpha}$ is the vibronic coupling constant (VCC) defined as follows:

$$
V_{i j, \alpha}=\left\langle\Psi_{i}\left(\mathbf{r} ; \mathbf{R}_{\mathbf{0}}\right)\left|\left(\frac{\partial \widehat{H}(\mathbf{r}, \mathbf{R})}{\partial Q_{\alpha}}\right)_{\mathbf{R}_{0}}\right| \Psi_{j}\left(\mathbf{r} ; \mathbf{R}_{\mathbf{0}}\right)\right\rangle,
$$

where $\widehat{H}(\mathbf{r}, \mathbf{R})$ is the molecular Hamiltonian, and $\mathbf{R}$ is a set of nuclear configuration. $V_{i j, \alpha}$ with $i \neq j$ is called the off-diagonal VCC, and $V_{j, \alpha}:=V_{j j, \alpha}$ is called the diagonal VCC.

In the following, we assume the displaced harmonic oscillator ignoring the Duschinsky effect, i.e. $\omega_{i, \alpha}=\omega_{j, \alpha}$ where $\omega_{i, \alpha}$ and $\omega_{j, \alpha}$ are respectively the angular frequencies of the initial and final vibrational modes. Then, the matrix element of the vibrational states in Eq.(S1) is expressed as ${ }^{11}$

$$
\left\langle\chi_{i m}(\mathbf{Q})\left|Q_{\alpha}\right| \chi_{j n}(\mathbf{Q})\right\rangle=\left\langle n_{i m, \alpha}\left|Q_{\alpha}\right| n_{j n, \alpha}\right\rangle \prod_{\beta \neq \alpha}\left\langle n_{i m, \beta} \mid n_{j n, \beta}\right\rangle,
$$

where $\left|n_{i m, \alpha}\left(Q_{\alpha}\right)\right\rangle$ denotes a vibrational state of mode $\alpha$ represented by its vibrational quantum number $n_{i m, \alpha}$. The matrix element of nuclear moment is given by

$$
\left\langle n_{i m, \alpha}\left|Q_{\alpha}\right| n_{j n, \alpha}\right\rangle=\sqrt{\frac{\hbar}{2 \omega_{j, \alpha}}}\left(\sqrt{n_{j n, \alpha}+1}\left\langle n_{i m, \alpha} \mid n_{j n, \alpha}+1\right\rangle+\sqrt{n_{j n, \alpha}}\left\langle n_{i m, \alpha} \mid n_{j n, \alpha}-1\right\rangle\right),
$$

and the Franck-Condon overlap integral is ${ }^{12}$

$$
\left\langle n_{i m, \alpha} \mid n_{j n, \alpha}\right\rangle=\sqrt{\frac{n_{i m, \alpha} ! n_{j n, \alpha} !}{2^{n_{i m, \alpha}+n_{j n, \alpha}}}} e^{-\frac{1}{4} g_{j, \alpha}^{2}} \sum_{l=0}^{\min \left[n_{i m, \alpha}, n_{j n, \alpha}\right]}(-1)^{n_{i m, \alpha}-l} 2^{l} \frac{g_{j, \alpha}^{n_{i m, \alpha}+n_{j n, \alpha}-2 l}}{l !\left(n_{i m, \alpha}-l\right) !\left(n_{j n, \alpha}-l\right) !} .
$$

Here, $g_{j, \alpha}$ is the dimensionless diagonal VCC expressed as

$$
g_{j, \alpha}=\frac{V_{j, \alpha}}{\sqrt{\hbar \omega_{j, \alpha}^{3}}}
$$

which corresponds to the geometrical displacement between electronic states $i$ and $j$ along the direction of mode $\alpha$. The matrix element of nuclear moment is simply referred as nuclear moment in the main text. 
$E_{i m}$ and $E_{j n}$ in Eq.(S1) are respectively the eigenvalues of the initial and final vibronic states, which consist of electronic and vibrational energies

$$
\begin{aligned}
& E_{i m}=E_{i}+\sum_{\alpha}\left(n_{i m, \alpha}+\frac{1}{2}\right) \hbar \omega_{i, \alpha}, \\
& E_{j n}=E_{j}+\sum_{\alpha}\left(n_{j n, \alpha}+\frac{1}{2}\right) \hbar \omega_{j, \alpha} .
\end{aligned}
$$

The electronic transition energy is defined as follows:

$$
E_{i j}=E_{i}-E_{j}
$$

It should be noted that the quantities required for the calculations of $k_{i \rightarrow j}^{\mathrm{lC}}$ are $V_{i j, \alpha}, \omega_{j, \alpha}, g_{j, \alpha}$, and $E_{i j}$.

In this study, we evaluated $k_{i \rightarrow j}^{\mathrm{IC}}$ within the effective mode approximation at $T=0 \mathrm{~K}$; that is

$$
k_{i \rightarrow j}^{\mathrm{lC}}=\frac{2 \pi}{\hbar} \sum_{m n} P_{i m}(T)\left|V_{i j, \max }\right|^{2}\left|\left\langle n_{i m, \xi}\left|Q_{\xi}\right| n_{j n, \xi}\right\rangle\right|^{2} L\left(E_{i m}^{\xi}-E_{j n}^{\xi}\right) .
$$

The off-diagonal VCCs are represented as the one for a maximum coupling mode, $V_{i j \text {,max }}$, because the main paths of internal conversion are via the vibrational modes that give the large off-diagonal VCCs. The nuclear moment is evaluated from the angular frequency and dimensionless diagonal VCC of an effective mode. The effective mode is defined by ${ }^{13}$

$$
\boldsymbol{u}_{j, \xi}=\sum_{\alpha} \frac{V_{j, \alpha}}{V_{j, \xi}} \boldsymbol{u}_{j, \alpha}
$$

where $\boldsymbol{u}_{j, \alpha}$ is the vibrational mode of mode $\alpha$, and $V_{j, \xi}$ denotes the VCC along the effective mode,

$$
V_{j, \xi}=\sqrt{\sum_{\alpha}\left|V_{j, \alpha}\right|^{2}} .
$$

The effective mode corresponds to the steepest descent direction to the minimum point on the energy surface of electronic state $j$. The angular frequency of the effective mode is given by

$$
\omega_{j, \xi}=\frac{\sqrt{\sum_{\alpha}\left|\omega_{j, \alpha} V_{j, \alpha}\right|^{2}}}{V_{j, \xi}},
$$

and, therefore, the dimensionless diagonal VCC of the effective mode is defined as follows:

$$
g_{j, \xi}=\frac{V_{j, \xi}}{\sqrt{\hbar \omega_{j, \xi}^{3}}} .
$$

The vibronic energy difference is given by

$$
E_{i m}^{\xi}-E_{j n}^{\xi}=E_{i j}+\left(n_{i m, \xi}-n_{j n, \xi}\right) \hbar \omega_{j, \xi},
$$

where $n_{i m, \xi}$ is the vibrational quantum number of the effective mode. It should be noted that we used the Lorentzian function with broadening factor $\gamma$ in Eq.(S10) instead of the delta function in Eq. (S1) 


$$
L(E)=\frac{1}{\pi} \frac{\gamma}{E^{2}+\gamma^{2}}
$$

for describing the density of final vibronic states. We fixed $\gamma$ to be $0.3 \mathrm{~cm}^{-1}$ in the calculations of $k_{i \rightarrow j}^{\mathrm{lC}}$ for PyBTM, bisPyTM, and trisPyM.

\section{Vibronic Coupling Constants of PyBTM, bisPyTM, and trisPyM}
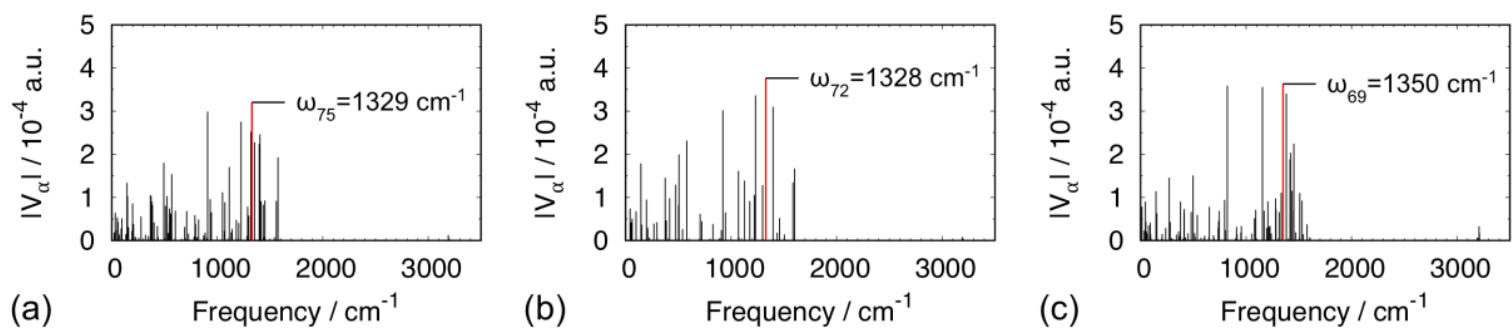

Figure S1. Off-diagonal VCCs of $\mathrm{D}_{1} @ \mathrm{D}_{1} \rightarrow \mathrm{D}_{0} @ \mathrm{D}_{1}$ for (a) PyBTM, (b) bisPyTM, and (c) trisPyM. The offdiagonal VCC of the maximum coupling mode is colored with red.
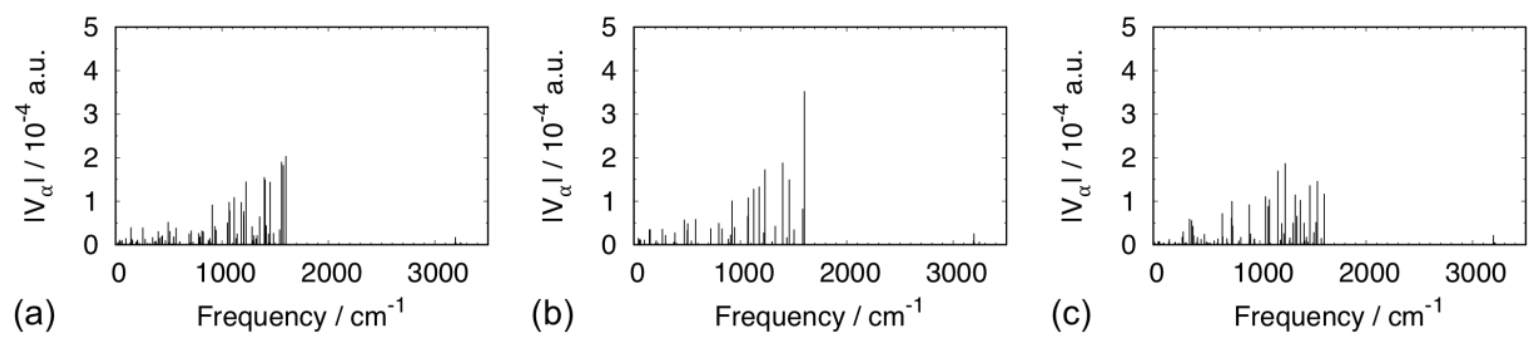

Figure S2. Diagonal VCCs of $\mathrm{D}_{0} @ \mathrm{D}_{1}$ for (a) PyBTM, (b) bisPyTM, and (c) trisPyM.

Table S1 Square of off-diagonal VCC for the maximum coupling mode, nuclear moment for the effective mode, density of final states, and their product when $n_{i m, \xi}=0$ and $n_{j n, \xi}=2$. The vibrational quantum numbers of $n_{i m, \xi}=0$ and $n_{j n, \xi}=2$ are the main contributions in $k_{i \rightarrow j}^{\mathrm{lC}}$.

\begin{tabular}{|c|c|c|c|c|}
\hline & $\begin{array}{c}\left|V_{i j, \mathrm{max}}\right|^{2} \\
/ 10^{-7} \text { a.u. }\end{array}$ & $\begin{array}{c}\left|\left\langle n_{i m, \xi}\left|Q_{\xi}\right| n_{j n, \xi}\right\rangle\right|^{2} \\
/ 10^{-1} \text { a.u. }\end{array}$ & $\begin{array}{r}L\left(E_{i m}^{\xi}-E_{j n}^{\xi}\right) \\
/ 10^{-5} \text { a.u. }\end{array}$ & $\left.\frac{2 \pi}{\hbar}\left|V_{i j, \max }\right|\right|^{2}\left|\left\langle n_{i m, \xi}\left|Q_{\xi}\right| n_{j n, \xi}\right\rangle\right|^{2} L\left(E_{i m}^{\xi}-E_{j n}^{\xi}\right)$ \\
\hline PyBTM & 1.0265 & 2.5763 & 8.1516 & 0.5600 \\
\hline bisPyTM & 1.4177 & 2.5278 & 9.0623 & 0.8436 \\
\hline trisPyM & 1.3176 & 2.7246 & 9.4581 & 0.8820 \\
\hline
\end{tabular}




\section{Vibronic Coupling Density}

The VCC is expressed as an integral of the spatially distributed density, $\eta_{i j, \alpha}(\boldsymbol{x})$, with a three-dimensional coordinate $\boldsymbol{x}=(x, y, z):^{14}$

$$
V_{i j, \alpha}=\int \eta_{i j, \alpha}(\boldsymbol{x}) d \boldsymbol{x}
$$

$\eta_{i j, \alpha}(\boldsymbol{x})$ is called the vibrionic coupling density (VCD). ${ }^{13}$ The off-diagonal VCD is defined by the product of the overlap density $\rho_{i j}(\boldsymbol{x})$ and potential derivative $v_{\alpha}(\boldsymbol{x})$

$$
\eta_{i j, \alpha}(\boldsymbol{x})=\rho_{i j}(\boldsymbol{x}) \times v_{\alpha}(\boldsymbol{x}),
$$

whereas the diagonal VCD, $\eta_{j, \alpha}:=\eta_{j j, \alpha}$, is defined by the product of the electron density difference $\Delta \rho_{i j}(\boldsymbol{x})$ and potential derivative $v_{\alpha}(\boldsymbol{x})$

$$
\eta_{j, \alpha}(\boldsymbol{x})=\Delta \rho_{i j}(\boldsymbol{x}) \times v_{\alpha}(\boldsymbol{x}) .
$$

Here, $v_{\alpha}(\boldsymbol{x})$ is the derivative of the nuclear-electron attraction potential $u_{e n}(\boldsymbol{x})$ acting on a single electron with respect to $Q_{\alpha}$ :

$$
v_{\alpha}(\boldsymbol{x})=\left(\frac{\partial u_{e n}(\boldsymbol{x})}{\partial Q_{\alpha}}\right)_{\boldsymbol{R}_{0}}
$$

$\rho_{i j}(\boldsymbol{x})$ is given by

$$
\rho_{i j}(\boldsymbol{x})=\left\langle\Psi_{i}\left(\mathbf{r} ; \mathbf{R}_{\mathbf{0}}\right)|\hat{\rho}(\boldsymbol{x})| \Psi_{j}\left(\mathbf{r} ; \mathbf{R}_{\mathbf{0}}\right)\right\rangle,
$$

where $\hat{\rho}(\boldsymbol{x})$ denotes the density operator defined by the field operators of a fermion:

$$
\hat{\rho}(\boldsymbol{x})=\hat{\psi}^{\dagger}(\boldsymbol{x}) \hat{\psi}(\boldsymbol{x}) .
$$

$\Delta \rho_{j}(\boldsymbol{x})$ is the electron-density difference between the electronic state $\left|\Psi_{i}\left(\mathbf{r} ; \mathbf{R}_{\mathbf{0}}\right)\right\rangle$ and $\mid \Psi_{j}\left(\mathbf{r} ; \mathbf{R}_{\mathbf{0}}\right)$ :

$$
\Delta \rho_{i j}(\boldsymbol{x})=\left\langle\Psi_{j}\left(\mathbf{r} ; \mathbf{R}_{\mathbf{0}}\right)|\hat{\rho}(\boldsymbol{x})| \Psi_{j}\left(\mathbf{r} ; \mathbf{R}_{\mathbf{0}}\right)\right\rangle-\left\langle\Psi_{i}\left(\mathbf{r} ; \mathbf{R}_{\mathbf{0}}\right)|\hat{\rho}(\boldsymbol{x})| \Psi_{i}\left(\mathbf{r} ; \mathbf{R}_{\mathbf{0}}\right)\right\rangle .
$$


MODE 75

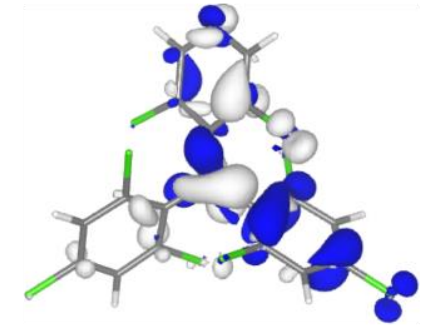

(a)

$$
\rho_{i j}
$$

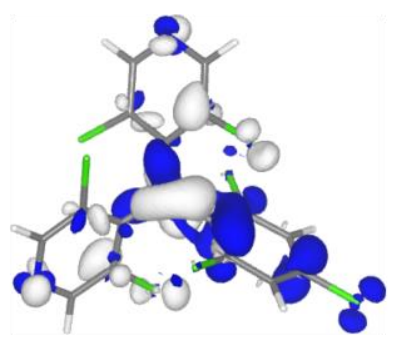

(b)

$$
\rho_{\mathrm{ij}}
$$

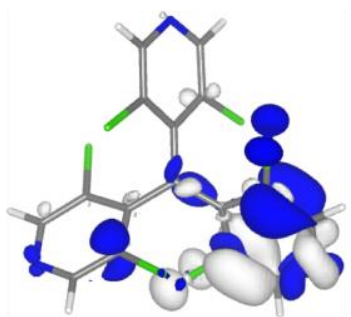

(c)

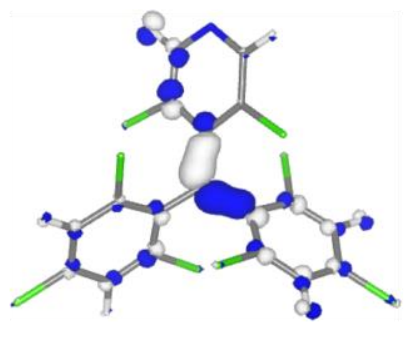

$\mathrm{v}_{\alpha}$

MODE 72

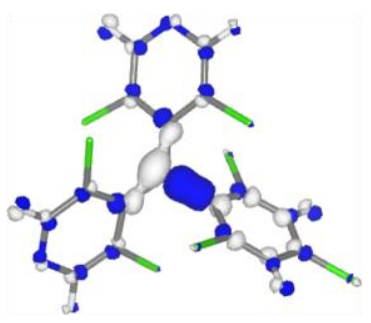

$\mathrm{v}_{\alpha}$

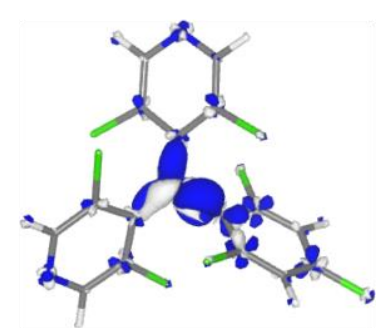

MODE 69

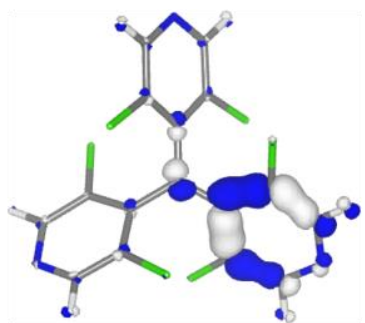

$\mathrm{v}_{\alpha}$ $\eta_{\mathrm{ij}, \alpha}$

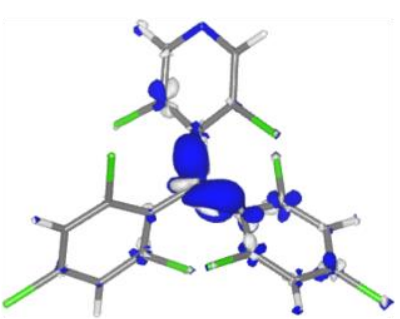

$\eta_{\mathrm{ij}, \alpha}$

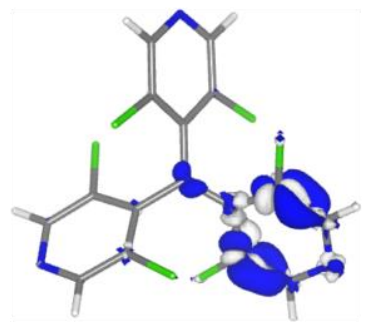

$\eta_{i j, \alpha}$

Figure S3. Off-diagonal VCD analyses of $\mathrm{D}_{1} @ \mathrm{D}_{1} \rightarrow \mathrm{D}_{0} @ \mathrm{D}_{1}$ for the maximum coupling mode of (a) PyBTM, (b) bisPyTM, and (c) trisPyM. Isosurface values of $\rho_{i j}, v_{\alpha}$, and $\eta_{i j, \alpha}$ are $1.0 \times 10^{-3}, 1.0 \times 10^{-2}$, and $1.0 \times 10^{-5}$ a.u., respectively. 


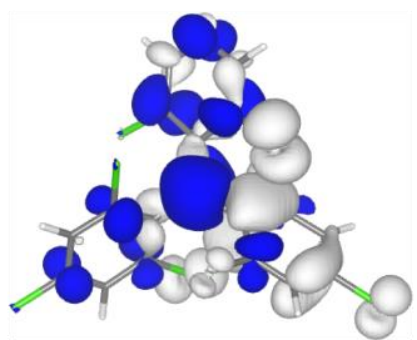

(a)
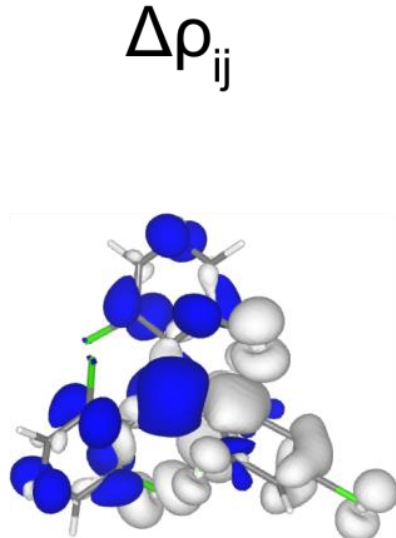

(b)
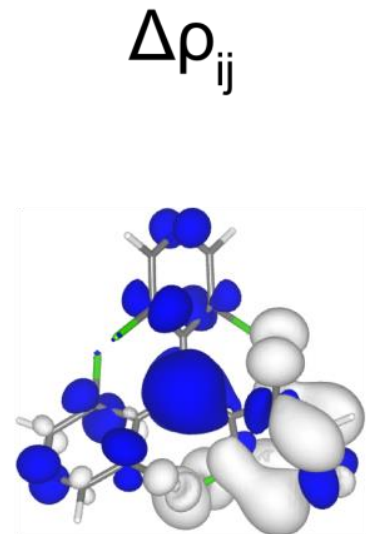

(c)

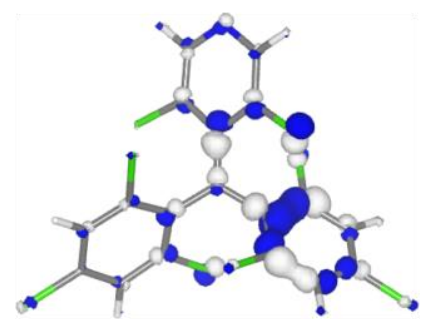

$\mathrm{v}_{\xi}$

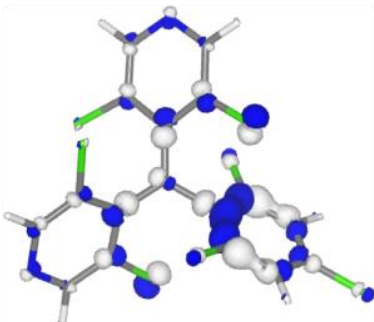

$\mathrm{V}_{\xi}$

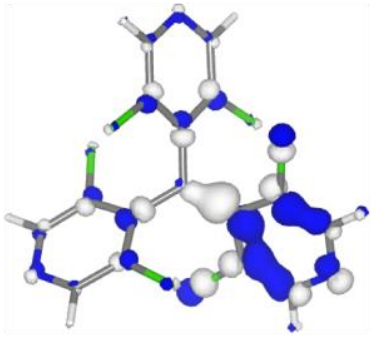

$\mathrm{v}_{\xi}$

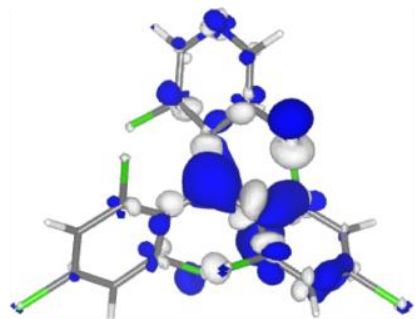

$\eta_{j, \xi}$

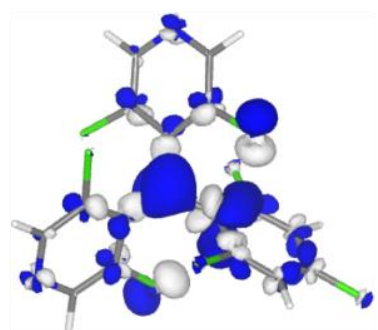

$\eta_{j, \xi}$

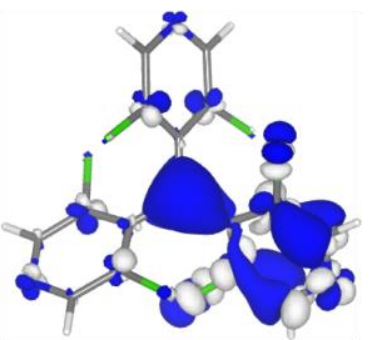

$\eta_{j, \xi}$

Figure S4. Diagonal VCD analyses of $\mathrm{D}_{0} @ \mathrm{D}_{1}$ for the effective mode of (a) PyBTM, (b) bisPyTM, and (c) trisPyM. Isosurface values of $\Delta \rho_{i j}, v_{\xi}$, and $\eta_{j, \xi}$ are $1.0 \times 10^{-3}, 1.0 \times 10^{-2}$, and $1.0 \times 10^{-5}$ a.u., respectively. 
Table S1 Hyperfine coupling constants (HCCs) used for ESR simulation and DFT-calculated values

\begin{tabular}{cccccc}
\hline hcc / mT & $\mathrm{N}$ & $\mathrm{H}$ & $\alpha^{-13} \mathrm{C}$ & ipso- ${ }^{13} \mathrm{C}$ & ortho- ${ }^{13} \mathrm{C}$ \\
\hline Sim. & 0.12 & 0.101 & 3.64 & 1.305 & 1.305 \\
\hline Calcd. & 0.1126 & 0.1404 & 3.8238 & 1.174 & 1.117 \\
& 0.1126 & 0.1404 & & 1.174 & 1.118 \\
& 0.1126 & 0.1405 & & 1.174 & 1.118 \\
& & 0.1405 & & & 1.118 \\
& & 0.1405 & & & 1.118 \\
& & 0.1405 & & & 1.118 \\
\hline
\end{tabular}

The other parameters used for the simulation was as follows: $g$ value $=2.00329$, Line width $=0.3 \mathrm{G}$, spectral shape $=100 \%$ Lorentzian.

Table S2 Absolute emission quantum yields and luminescent lifetimes of trisPyM in various solvents

\begin{tabular}{ccc}
\hline & Quantum yield / \% & lifetime / ns \\
\hline$n$-hexane & 0.86 & 3.46 \\
$\mathrm{CH}_{2} \mathrm{Cl}_{2}$ & 0.85 & 3.00 \\
$\mathrm{CHCl}_{3}$ & 0.85 & 2.97 \\
Ethyl acetate & 0.64 & 2.26 \\
Acetonitrile & 0.60 & 2.05 \\
$\mathrm{Et}_{2} \mathrm{O}$ & 0.41 & - \\
Acetone & 0.37 & - \\
THF & 0.26 & - \\
Methanol & 0.23 & - \\
Ethanol & 0.22 & - \\
Toluene & 0.22 & - \\
\hline
\end{tabular}

Excitation wavelengths were $350 \mathrm{~nm}$ for all solutions (ca. $\left.5 \times 10^{6} \mathrm{M}\right)$.

Luminescent lifetimes were measured at $700 \mathrm{~nm}$ for all solutions. 
Table S3 Half-lives $\left(t_{1 / 2}\right)$ and absorption coefficients $(\varepsilon)$ of trisPyM and comparisons of photostability

\begin{tabular}{cccc}
\hline & Half-lives $\left(t_{1 / 2}\right) / \mathrm{s}^{\mathrm{a}}$ & $\varepsilon / 10^{4} \mathrm{M}^{-1} \mathrm{~cm}^{-1}$ & $\begin{array}{c}\varepsilon \times t_{1 / 2} \text { for } \\
\text { trisPyM/bisPyTM }\end{array}$ \\
\hline$n$-hexane & $2.38(8) \times 10^{4}$ & 1.93 & 3.7 \\
$\mathrm{CH}_{2} \mathrm{Cl}_{2}$ & $2.22(6) \times 10^{4}$ & 1.90 & 4.1 \\
$\mathrm{CHCl}_{3}$ & $6.9(2) \times 10^{3}$ & 1.90 & 1.3 \\
Acetonitrile & $2.4(1) \times 10^{4}$ & 1.83 & 1.7 \\
Acetone & $2.59(1) \times 10^{3}$ & 1.74 & 1.0 \\
Methanol & $2.8(3) \times 10$ & 1.76 & 2.6 \\
Ethanol & $1.7(1) \times 10$ & 1.67 & 1.2 \\
\hline
\end{tabular}

a Excitation wavelengths were $350 \mathrm{~nm}$ for all solutions (ca. $5 \times 10^{6} \mathrm{M}$ ).

${ }^{b}$ Calculated based on the values in the previous report ${ }^{2}$ scaled with $t_{1 / 2}$ value of bisPyTM measured in this study $\left(5.7 \times 10^{3}\right)$.

Table S4 Photophysical parameters of radicals in $\mathrm{CH}_{2} \mathrm{Cl}_{2}$

\begin{tabular}{cccccccc}
\hline Radical & $\lambda_{\mathrm{em}} / \mathrm{nm}$ & $\lambda_{\mathrm{abs} 1^{\mathrm{a}}} / \mathrm{nm}$ & $\lambda_{\mathrm{abs} 2^{\mathrm{b}} / \mathrm{nm}}$ & $\phi_{\mathrm{em}}$ & $\tau / \mathrm{ns}$ & $k_{\mathrm{r}} / \times 10^{6} \mathrm{~s}^{-1}$ & $k_{\mathrm{nr}} / \times 10^{8} \mathrm{~s}^{-1}$ \\
\hline trisPyM & 700 & 518 & 350 & 0.0085 & 3.0 & 2.8 & 3.3 \\
bisPyTM $^{2}$ & 650 & 536 & 355 & 0.009 & 3.6 & 2.5 & 2.8 \\
PyBTM $^{1}$ & 585 & 541 & 370 & 0.025 & 7.4 & 3.9 & 1.5 \\
TTM $^{1,2}$ & 570 & 542 & 373 & 0.02 & 7.0 & 3 & 1.4 \\
\hline
\end{tabular}

a Peaks of longest absorption bands.

b Peaks of strongest absorption bands (> $300 \mathrm{~nm}$ ). 
Table S5 Crystallographic data of trisPyM and trisZn

\begin{tabular}{|c|c|c|}
\hline & trisPyM & trisZn \\
\hline CCDC Number & 2041203 & 2048812 \\
\hline Empirical formula & $\mathrm{C}_{16} \mathrm{H}_{6} \mathrm{Cl}_{6} \mathrm{~N}_{3}$ & $\mathrm{C}_{31} \mathrm{H}_{9} \mathrm{Cl}_{6} \mathrm{Zn}_{1.5} \mathrm{~F}_{18} \mathrm{~N}_{3} \mathrm{O}_{6}$ \\
\hline $\mathrm{FW} / \mathrm{g} \mathrm{mol}^{-1}$ & 452.94 & 1172.17 \\
\hline Crystal system & Monoclinic & Triclinic \\
\hline Space group & $C 2 / \mathrm{c}$ & $P-1$ \\
\hline Crystal size / mm & $0.608 \times 0.565 \times 0.377$ & $0.57 \times 0.38 \times 0.13$ \\
\hline Temperature / K & 143 & 123 \\
\hline$a / \AA$ & $15.1551(4)$ & $9.2814(2)$ \\
\hline$b / \AA$ & $15.0765(3)$ & $14.6585(3)$ \\
\hline$c / \AA$ & $7.4458(2)$ & $20.5302(5)$ \\
\hline$\alpha /^{\circ}$ & 90 & $109.529(2)$ \\
\hline$\beta /{ }^{\circ}$ & $91.101(2)$ & $93.901(2)$ \\
\hline$\gamma /{ }^{\circ}$ & 90 & $97.730(2)$ \\
\hline$V / \AA^{3}$ & $1700.95(7)$ & $2589.39(10)$ \\
\hline$\rho_{\text {calced }} / \mathrm{g} \mathrm{cm}^{-3}$ & 1.769 & 1.503 \\
\hline$\lambda / \AA$ & 0.71073 & 0.71073 \\
\hline$\mu / \mathrm{mm}^{-1}$ & 1.015 & 1.112 \\
\hline Reflection collected & 11197 & 39983 \\
\hline Independent reflections & 1944 & 11858 \\
\hline Parameters & 115 & 595 \\
\hline $\mathrm{R}_{\text {int }}$ & 0.0267 & 0.0789 \\
\hline$R_{1}^{\mathrm{a}}$ & 0.0246 & 0.0615 \\
\hline$w R_{2}^{\mathrm{b}}$ & 0.0638 & 0.1945 \\
\hline $\mathrm{GoF}^{\mathrm{c}}$ & 1.082 & 1.033 \\
\hline
\end{tabular}

${ }^{a} R_{1}=\Sigma|| F^{\mathrm{o}}|-| F^{\mathrm{c}}|| / \Sigma\left|F^{\mathrm{o}}\right|(\mid>2 \sigma(\Lambda)) .{ }^{b} w R_{2}=\left[\Sigma\left(w\left(F^{\mathrm{o} 2}-F^{\mathrm{c} 2}\right)^{2} / \Sigma w\left(F^{02}\right)^{2}\right]^{1 / 2}(1>2 \sigma(I))\right.$.

${ }^{c} \mathrm{GoF}=\left[\Sigma\left(w\left(F^{\circ 2}-F^{\mathrm{c} 2}\right)^{2} / \Sigma\left(N^{N}-N^{p}\right)^{2}\right]\right.$. 


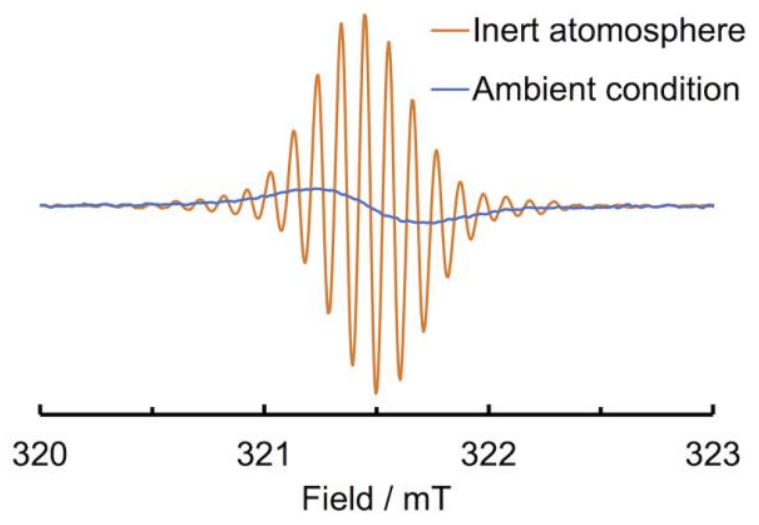

Figure S5. ESR signals of trisPyM in $\mathrm{CH}_{2} \mathrm{Cl}_{2}$ at room temperature after freeze-pump-thaw cycles (orange line) and after exposure to the air (blue line).

(a)

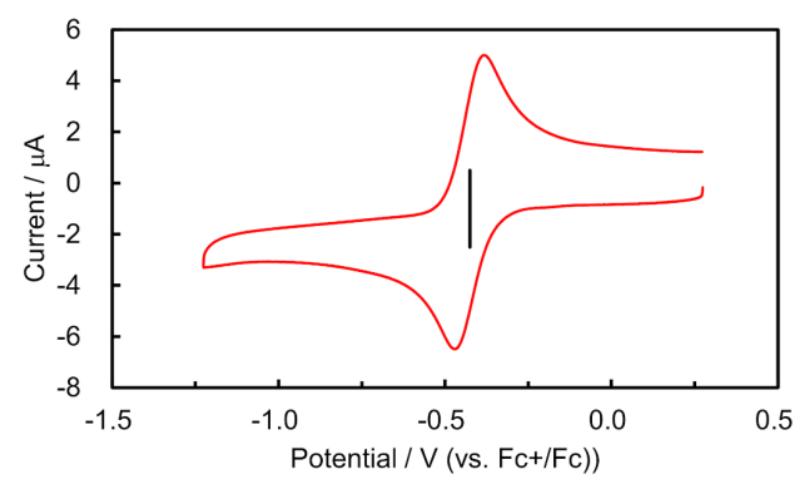

(b)

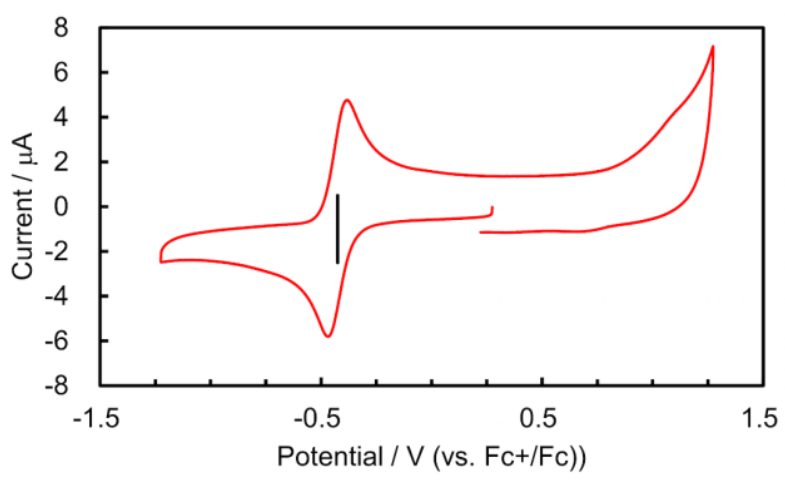

Figure S6. Cyclic voltammograms of trisPyM $(0.5 \mathrm{mM})$ in $0.1 \mathrm{M}^{n} \mathrm{Bu}_{4} \mathrm{NPF}_{6} / \mathrm{CH}_{2} \mathrm{Cl}_{2}$ at a scan rate of $0.1 \mathrm{~V}$ $\mathrm{s}^{-1}$ in the range of $(\mathrm{a})-1.23$ to $0.27 \mathrm{~V}$ and $(\mathrm{b})-1.23$ to $1.27 \mathrm{~V}$.

(a)

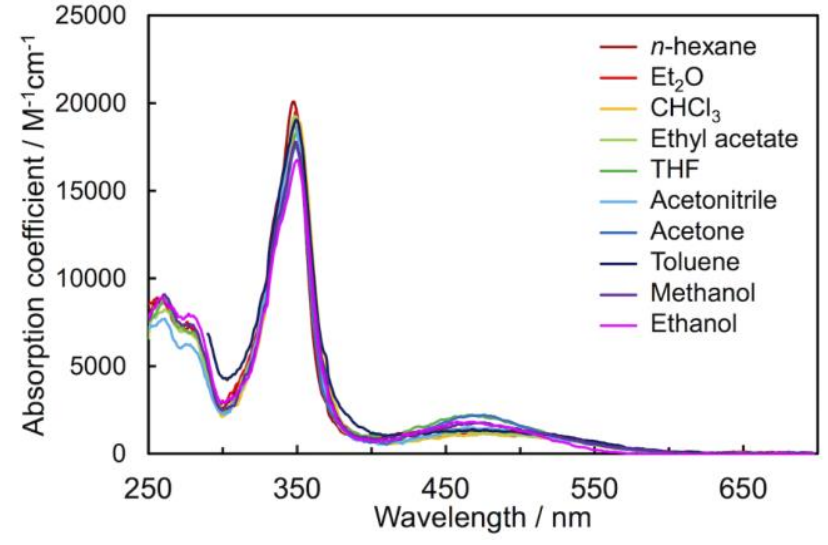

(b)

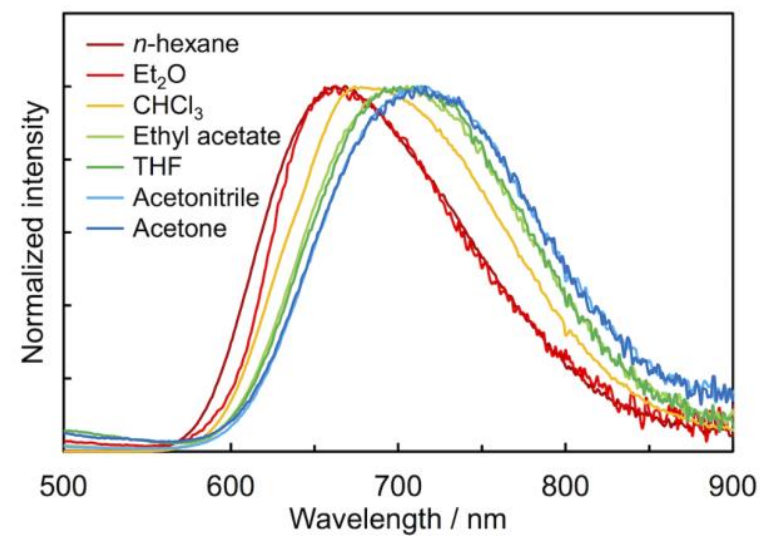

Figure S7. Absorption and emission spectra of trisPyM in various solvents. 


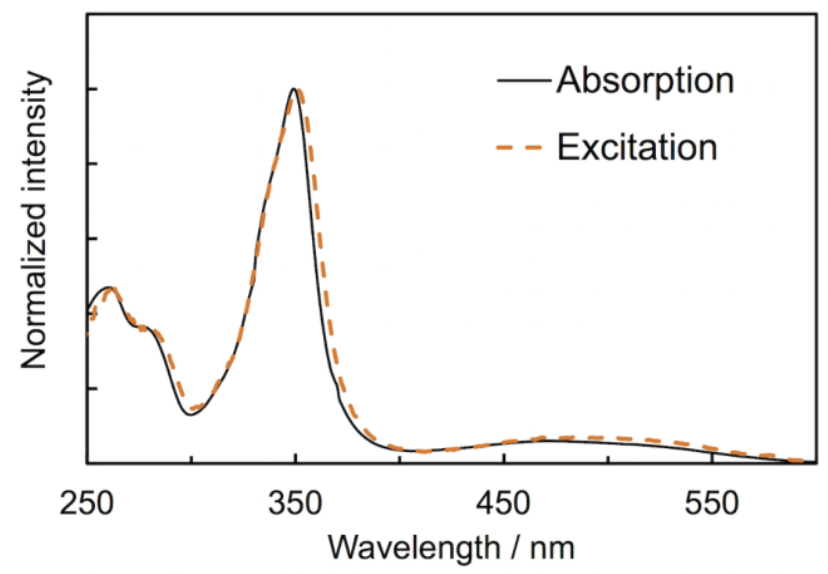

Figure S8. Excitation spectrum for emission wavelength at $700 \mathrm{~nm}$ and absorption spectrum of trisPyM in $\mathrm{CH}_{2} \mathrm{Cl}_{2}$.

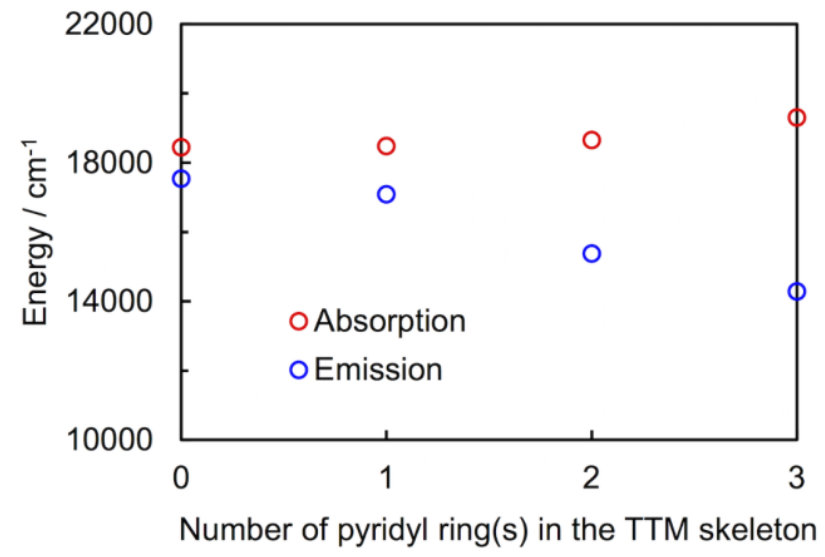

Figure S9. Energies of the longest absorption band peaks and the emission band peaks of radicals. 


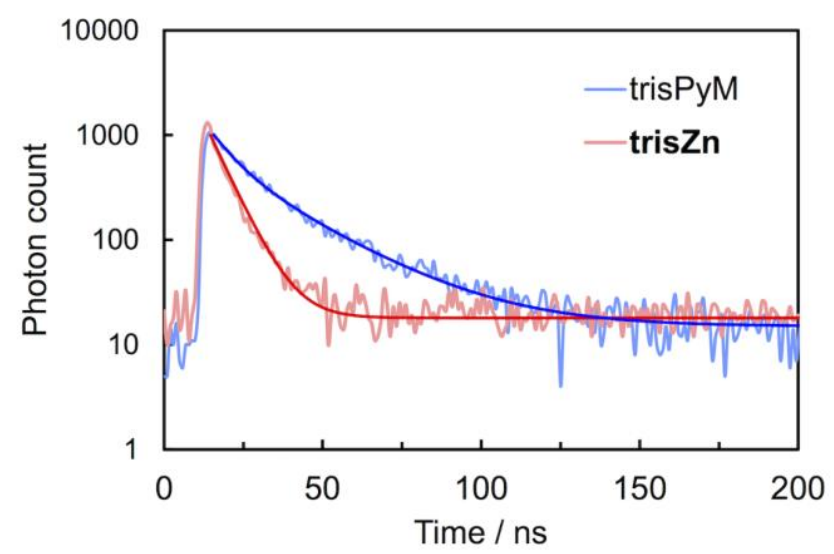

Figure S10. Emission decays of trisPyM $\left(\lambda_{\mathrm{em}}=665 \mathrm{~nm}\right)$ and trisZn $\left(\lambda_{\mathrm{em}}=695 \mathrm{~nm}\right)$ in the solid states at 79 $\mathrm{K}$ and the corresponding fitting curves. The decay curves of trisPyM and trisZn were fitted by the double and single exponential curves with the averaged lifetime of $20.0 \mathrm{~ns}\left(\tau_{1}=7.2 \mathrm{~ns}\left(\alpha_{1}=0.52\right), \tau_{2}=24.1 \mathrm{~ns}\left(\alpha_{2}\right.\right.$ $=0.48)$ ) and the lifetime of $6.6 \mathrm{~ns}$, respectively.

The average lifetime ( $\left.\tau_{\text {ave }}\right)$ was estimated with the Eq.(S24). ${ }^{15}$

$$
\tau_{\text {ave }}=\frac{\sum_{i} \alpha_{i} \tau_{i}^{2}}{\sum_{i} \alpha_{i} \tau_{i}}
$$

where $\alpha$ and $\tau$ are the pre-exponential factor and the lifetime of each component, respectively.

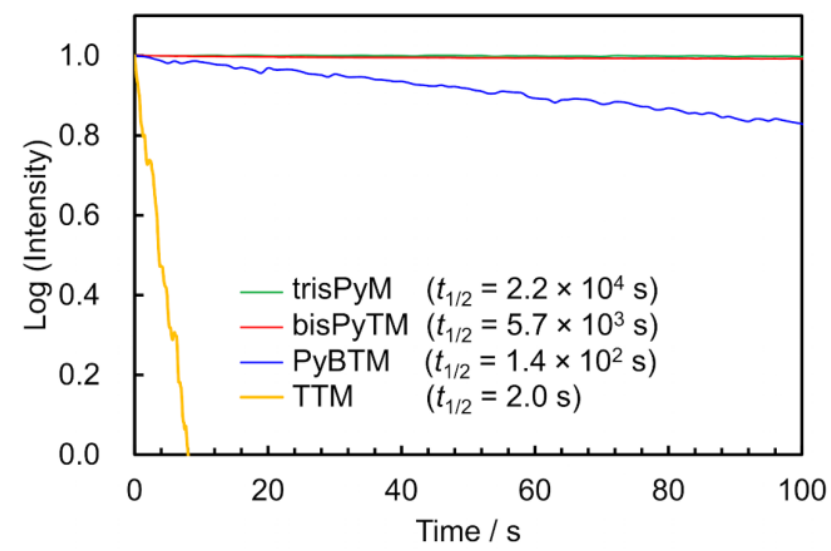

Figure S11. Enlarged figure of Figure 5. 


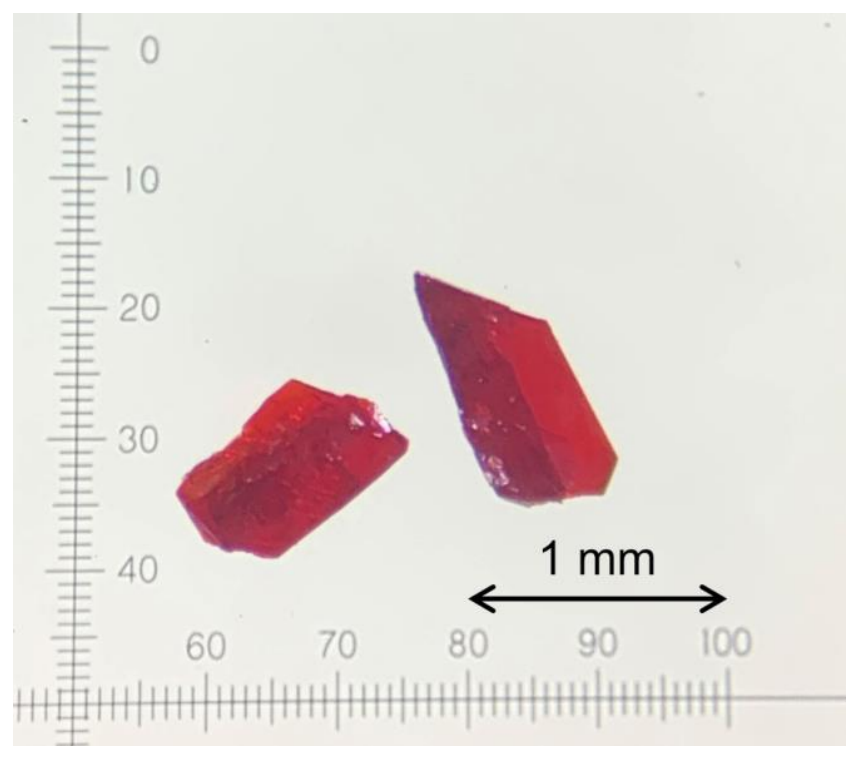

Figure S12. A photograph of crystals of trisZn.

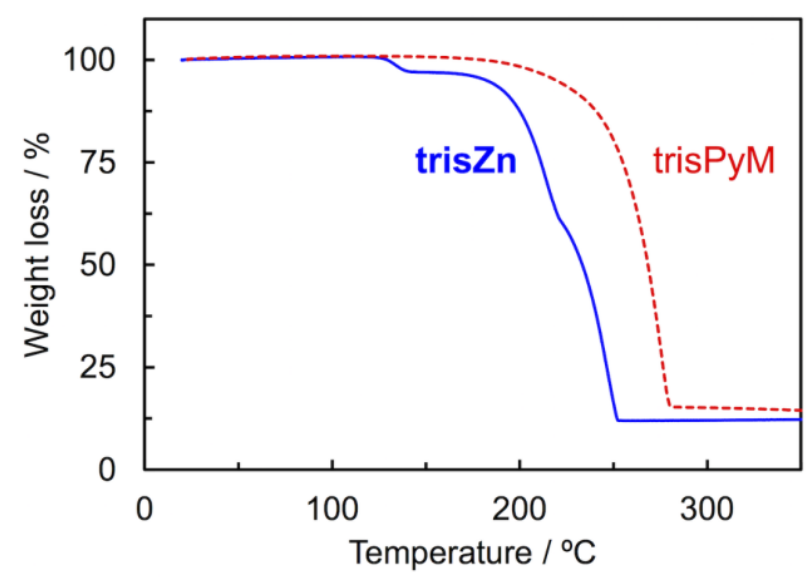

Figure S13. Thermogravimetric analysis (TGA) curves of trisZn and trisPyM under a scan rate of $2 \mathrm{~K}$ $\min ^{-1}$. 


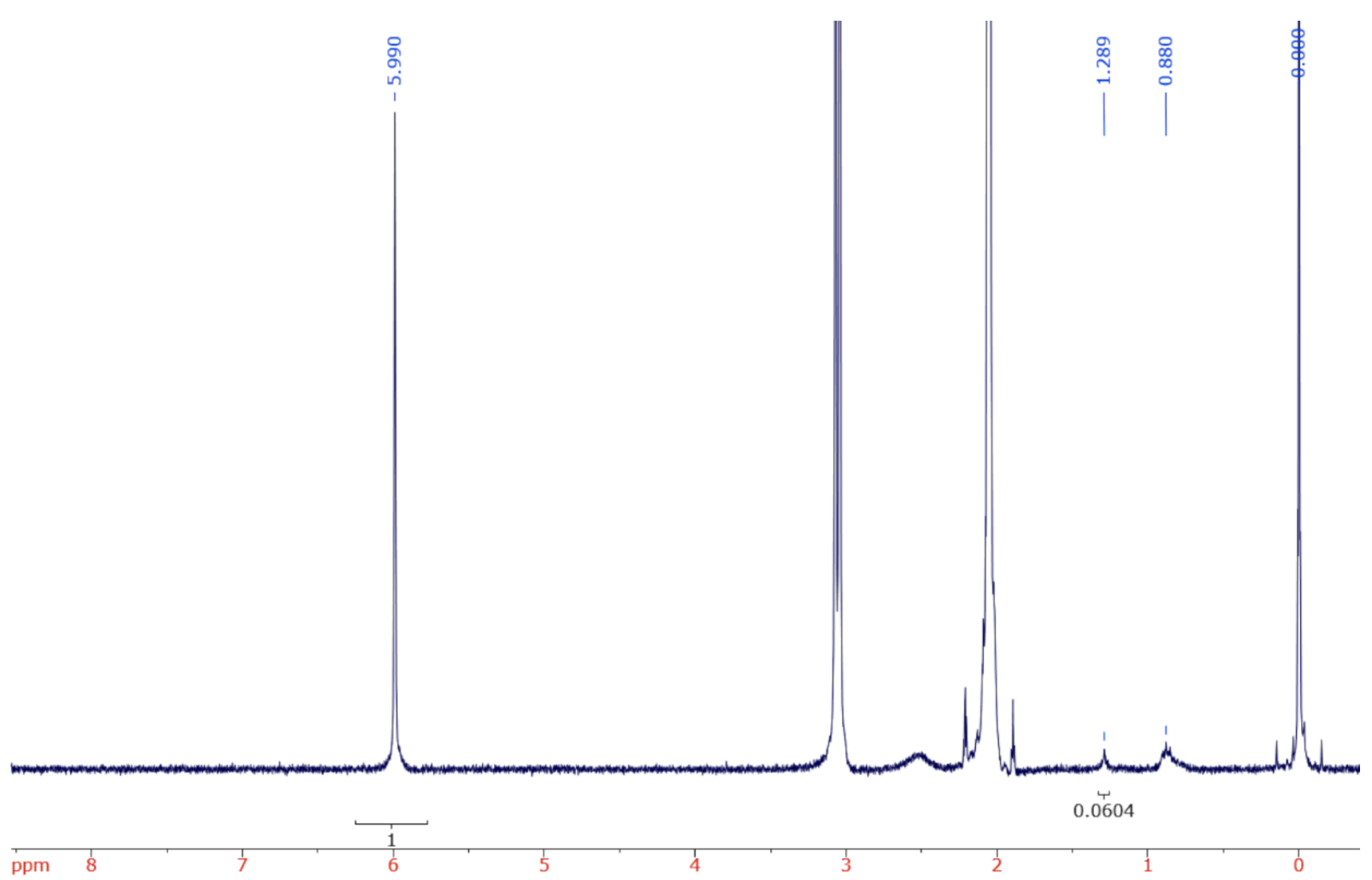

Figure S14. ${ }^{1} \mathrm{H}$ NMR spectra of trisZn in acetone-d6. Remaining $n$-hexane in tris $\mathbf{Z n}$ was calculated to be lower than $0.2 \mathrm{wt} \%$ based on the peak area ratio of hfac moieties of trisZn ( $\delta 5.99)$ and $n$-hexane $(\delta 1.29)$. We note that peaks $(\delta 0,88$ and 1.29) might be attributed to not $n$-hexane but grease. No peak attributed to remaining $\mathrm{CHCl}_{3}$ was observed and the broad peak around $\delta 2.5$ may be attributed to water molecules coordinated $\mathrm{Zn}$ " ions.

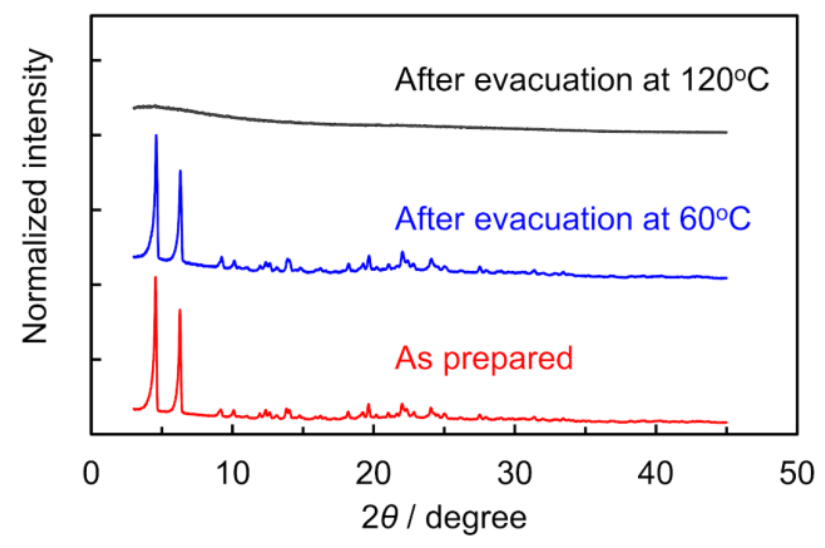

Figure S15. Powder X-ray diffraction patterns of trisZn for an as-prepared sample and samples after evacuation at $60^{\circ} \mathrm{C}$ for 12 hours and $120^{\circ} \mathrm{C}$ for 12 hours. 


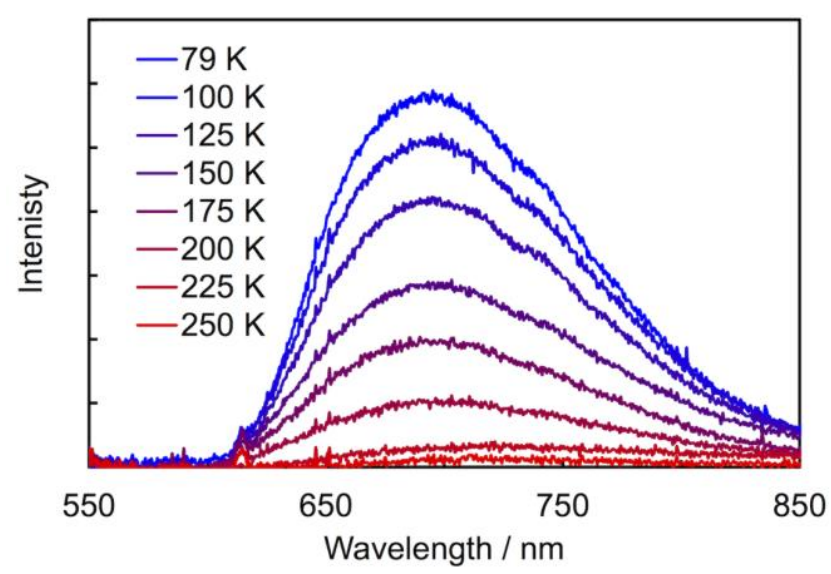

Figure S16. Temperature dependence of the emission spectra of trisZn $\left(\lambda_{\mathrm{ex}}=375 \mathrm{~nm}\right)$.

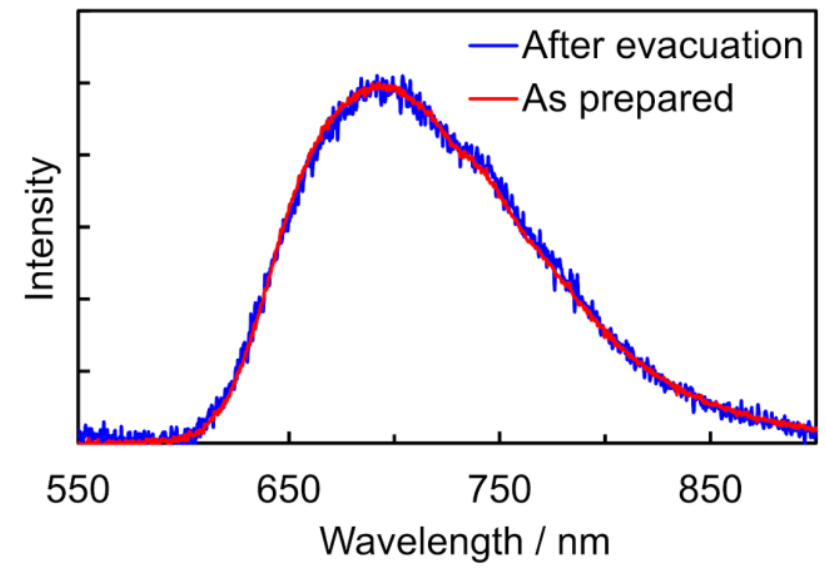

Figure S17. Emission spectra of trisZn at $79 \mathrm{~K}$ for the as-prepared sample and the sample after evacuation at $60^{\circ} \mathrm{C}$ for 12 hours.

(a)

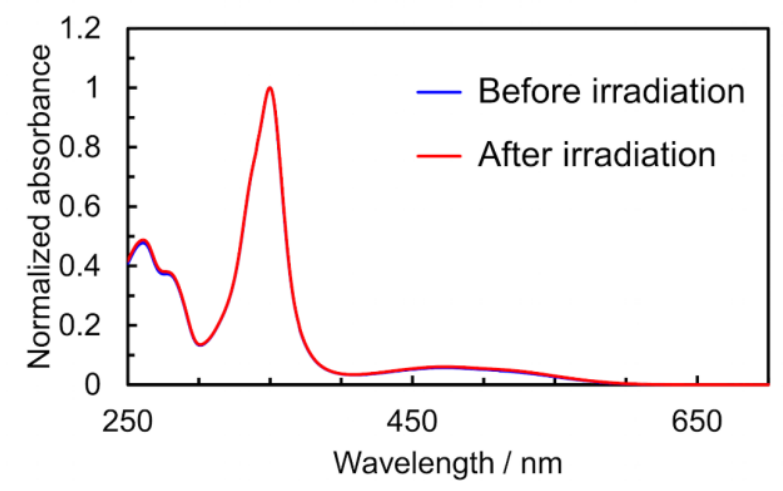

(b)

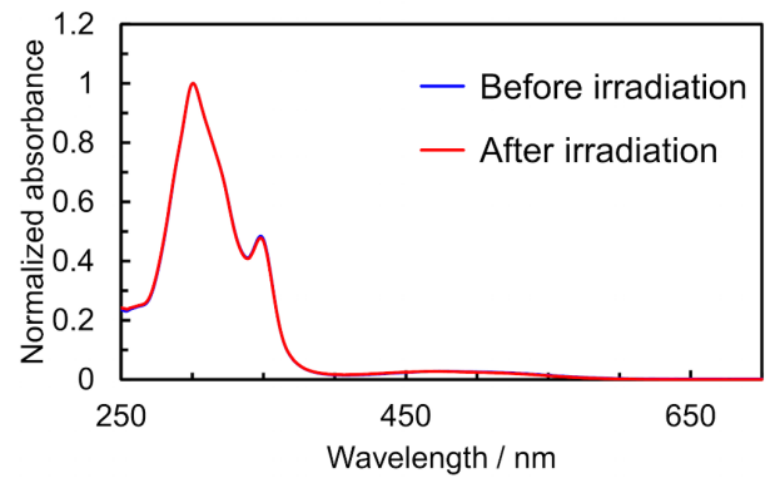

Figure S18. Absorption spectra of (a) trisPyM in $\mathrm{CH}_{2} \mathrm{Cl}_{2}$ and (b) trisZn in ethyl acetate. The samples were dissolved in the solvents for the measurements before and after continuous UV light irradiation ( $\lambda_{\mathrm{ex}}=350$ $\mathrm{nm}$ ) in the solid states. 
(a)

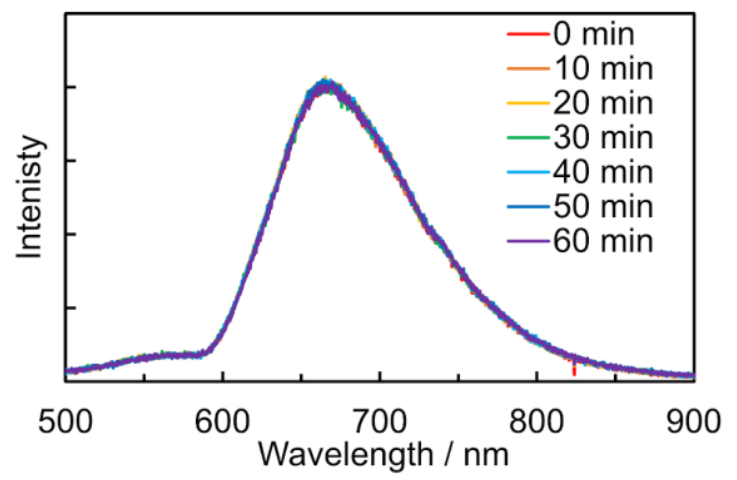

(c)

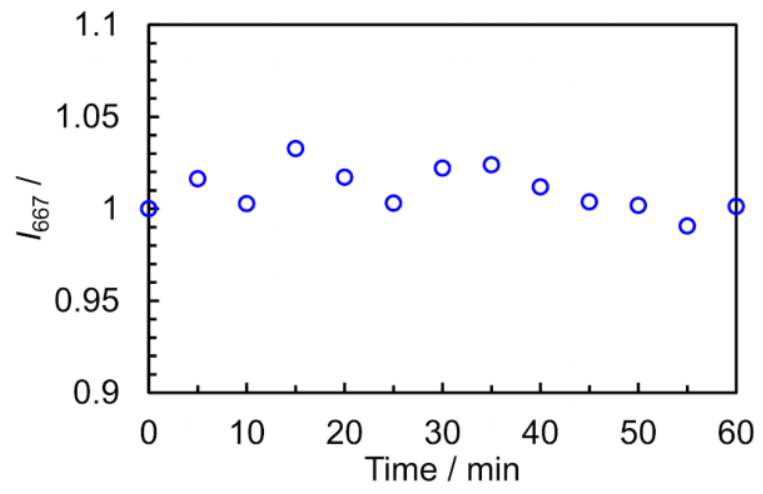

(b)

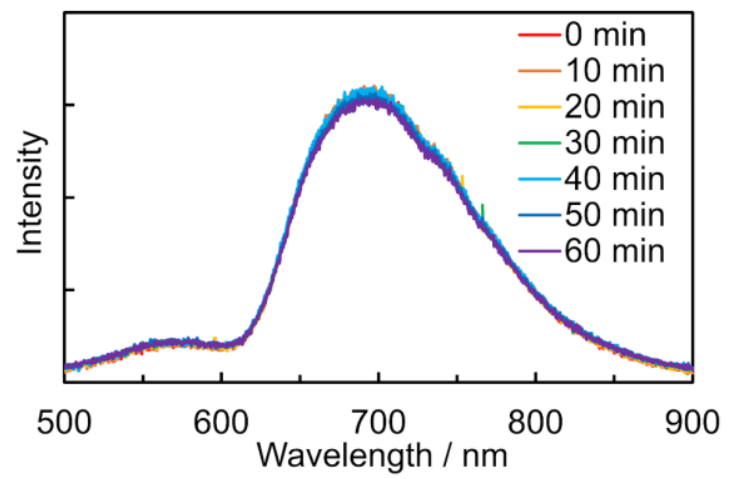

(d)

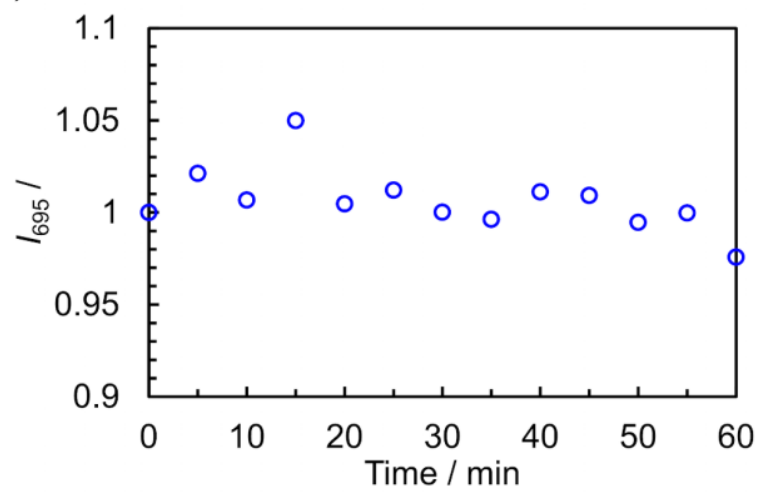

Figure S19. Emission spectra of (a) trisPyM and (b) trisZn and emission intensity of (c) trisPyM at $\lambda_{\mathrm{em}}=$ $667 \mathrm{~nm}$ and $(\mathrm{d})$ trisZn at $\lambda_{\mathrm{em}}=695 \mathrm{~nm}$ at $79 \mathrm{~K}$ under continuous excitation with a picosecond diode laser $\left(\lambda_{\mathrm{ex}}=375 \mathrm{~nm}\right)$.

(a)

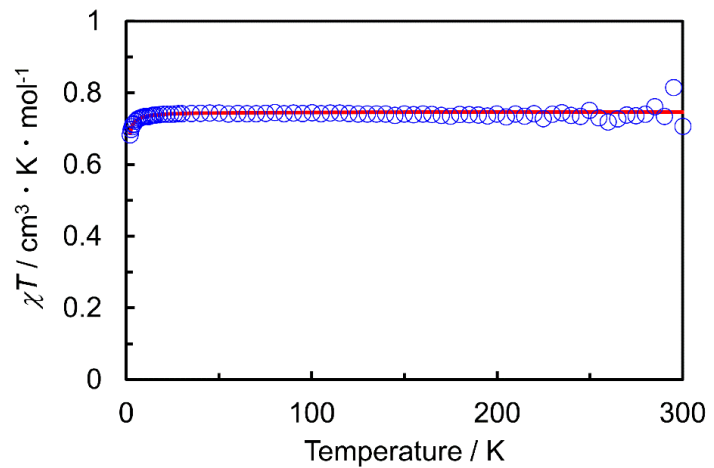

(b)

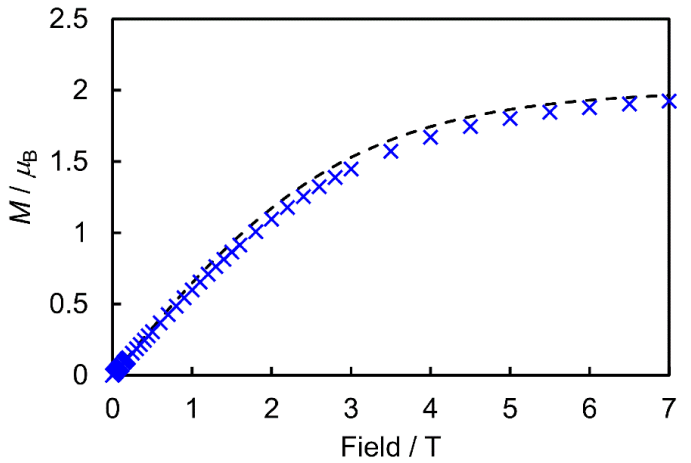

Figure S20. Magnetic properties of trisZn. (a) Temperature-dependent $\chi T$ under $1 \mathrm{~T}$. The red line indicates a fitting curve. (b) The magnetization vs. external magnetic field $(M-H)$ curve with doubled Brillouin function for $S=1 / 2$ (dashed line) at $2 \mathrm{~K} . \chi$ and magnetization per $\left[\mathrm{Zn}^{\prime \prime}(\mathrm{hfac})_{2}\right]_{3}[\operatorname{trisPyM}]_{2}$ unit were shown in the figures (a) and (b), respectively. 
(a)

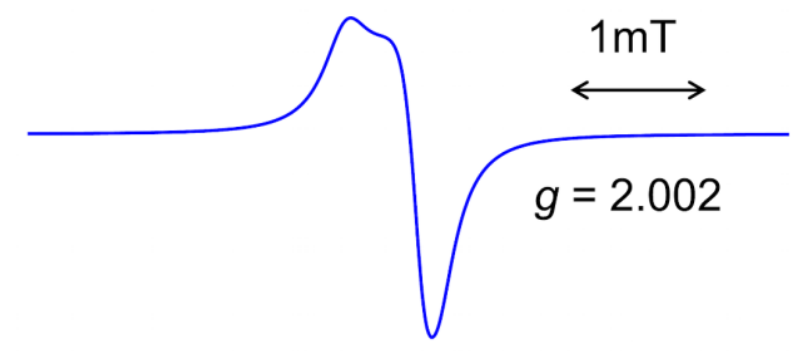

(b)

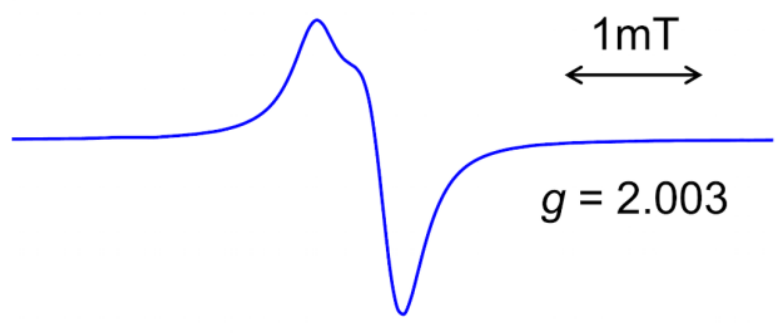

Figure S21. ESR signals of (a) trisPyM and (b) trisZn in the solid states at room temperature. 


\section{References}

1 Hattori, Y.; Kusamoto, T.; Nishihara, H. Luminescence, stability, and proton response of an open-shell (3,5dichloro-4-pyridyl)bis(2,4,6-trichlorophenyl)methyl radical. Angew. Chem. Int. Ed. 2014, 53 (44), 1184511848.

2 Kimura, S.; Tanushi, A.; Kusamoto, T.; Kochi, S.; Sato, T.; Nishihara, H. A Luminescent Organic Radical with Two Pyridyl Groups: High Photostability and Dual Stimuli-Responsive Properties, with Theoretical Analyses of Photophysical Processes. Chem. Sci. 2018, 9 (7), 1996-2007.

3 Sheldrick, G. M. SHELXT - Integrated space-group and crystal-structure determination. Acta Cryst. A, 2015, 71, 3-8.

4 Sheldrick, G. M. Crystal structure refinement with SHELXL. Acta Cryst. C, 2015, 71, 3-8.

5 Frisch, M. J.; Trucks, G. W.; Schlegel, H. B.; Scuseria, G. E.; Robb, M. A.; Cheeseman, J. R.; Scalmani, G.; Barone, V.; Petersson, G. A.; Nakatsuji, H.; Li, X.; Caricato, M.; Marenich, A. V.; Bloino, J.; Janesko, B. G.; Gomperts, R.; Mennucci, B.; Hratchian, H. P.; Ortiz, J. V.; Izmaylov, A. F.; Sonnenberg, J. L.; WilliamsYoung, D.; Ding, F.; Lipparini, F.; Egidi, F.; Goings, J.; Peng, B.; Petrone, A.; Henderson, T.; Ranasinghe, D.; Zakrzewski, V. G.; Gao, J.; Rega, N.; Zheng, G.; Liang, W.; Hada, M.; Ehara, M.; Toyota, K.; Fukuda, R.; Hasegawa, J.; Ishida, M.; Nakajima, T.; Honda, Y.; Kitao, O.; Nakai, H.; Vreven, T.; Throssell, K.; Montgomery, J. A., Jr.; Peralta, J. E.; Ogliaro, F.; Bearpark, M. J.; Heyd, J. J.; Brothers, E. N.; Kudin, K. N.; Staroverov, V. N.; Keith, T. A.; Kobayashi, R.; Normand, J.; Raghavachari, K.; Rendell, A. P.; Burant, J. C.; Iyengar, S. S.; Tomasi, J.; Cossi, M.; Millam, J. M.; Klene, M.; Adamo, C.; Cammi, R.; Ochterski, J. W.; Martin, R. L.; Morokuma, K.; Farkas, O.; Foresman, J. B.; Fox, D. J. Gaussian 16, Revision C.01, Gaussian, Inc., Wallingford CT, 2016.

6 Becke, A.D. Density-functional thermochemistry. III. The role of exact exchange. J. Chem. Phys. 1993, 98 (7), 5648-5652.

7 Hariharan, P. C.; Pople, J. A. The Influence of Polarization Functions on Molecular Orbital Hydrogenation Energies. The influence of polarization functions on molecular orbital hydrogenation energies. Theor. Chim. Acta 1973, 28, 213-222.

8 Tomasi, J.; Mennucci, B.; Cammi, R. Quantum Mechanical Continuum Solvation Models. Chem. Rev. 2005, 105 (8), 2999-3093.

9 Armet, O.; Veciana, J.; Rovira, C.; Riera, J.; Castañer, J.; Molins, E.; Rius, J.; Miravitlles, C.; Olivella, S.; Brichfeus, J. Inert Carbon Free Radicals. 8. Polychlorotriphenylmethyl Radicals. Synthesis, Structure, and Spin-Density Distribution. J. Phys. Chem. 1987, 91 (22), 5608-5616.

10 Fischer, G. Vibronic Coupling: The Interaction between the Electronic and Nuclear Motions, Academic Press; London, 1984.

11 Uejima, M.; Sato, T.; Yokoyama, D.; Tanaka, K.; Park, J.-W. Quantum yield in blue-emitting anthracene derivatives: vibronic coupling density and transition dipole moment density. Phys. Chem. Chem. Phys. 2014, 16, 14244-14256.

12 Hutchisson, E.; Band spectra intensities for symmetrical diatomic molecules. Phys. Rev. 1930, 36 (3), 410 420.

13 Sato, T.; Iwahara, N.; Haruta, N.; Tanaka, T. C60 bearing ethylene moieties. Chem. Phys. Lett. 2012, 531, 257-260. 
14 Sato, T.; Tokunaga, K.; Tanaka, K. Vibronic coupling in naphthalene anion: vibronic coupling density analysis for totally symmetric vibrational modes. J. Phys. Chem. A 2008, 112 (4), 758-767.

15 Lakowicz, J. R. Principles of Fluorescence Spectroscopy; Springer, 1999. 